\title{
Spin-Isospin Structure and Pion Condensation in Nucleon Matter
}

\author{
A. Akmalt and V. R. Pandharipanded \\ Department of Physics, University of Illinois at Urbana-Champaign, 1110 W. Green St., \\ Urbana, IL 61801
}

(May 25, 2022)

\begin{abstract}
We report variational calculations of symmetric nuclear matter and pure neutron matter, using the new Argonne $v_{18}$ two-nucleon and Urbana IX threenucleon interactions. At the equilibrium density of $0.16 \mathrm{fm}^{-3}$ the two-nucleon densities in symmetric nuclear matter exhibit a short-range spin-isospin structure similar to that found in light nuclei. We also find that both symmetric nuclear matter and pure neutron matter undergo transitions to phases with pion condensation at densities of $0.32 \mathrm{fm}^{-3}$ and $0.2 \mathrm{fm}^{-3}$, respectively. Neither transtion occurs with the Urbana $v_{14}$ two-nucleon interaction, while only the transition in neutron matter occurs with the Argonne $v_{14}$ two-nucleon interaction. The three-nucleon interaction is required for the transition to occur in symmetric nuclear matter, whereas the transition in pure neutron matter occurs even in its absence. The behavior of the isovector spin-longitudinal response and the pion excess in the vicinity of the transition, and the model dependence of the transition are discussed.
\end{abstract}

PACS numbers: 21.65.+f, 21.30.Fe, 26.60.+c, 64.70.-p 


\section{INTRODUCTION}

A central problem in nuclear many-body theory is the prediction from realistic models of nuclear forces, of properties of uniform nuclear matter in stable nuclei and in neutron stars. This problem has been the focus of much attention since the pioneering work of Brueckner, Levinson and Mahmoud [1] in 1954. Early work in this field, based primarily on Brueckner-Bethe-Goldstone many-body theory, was reviewed by Bethe [2] in 1971. A variational method based on hypernetted chain summation techniques (VCS, previously denoted FHNC-SOC) was developed in the 1970s [3], particularly to include the effects of many-body correlations, presumably important in dense neutron star matter. Calculations performed since then have confirmed that many-body clusters make significant contributions to the binding energies of equilibrium nuclear matter and light nuclei [3] 8 .

Friedman and Pandharipande [9] (hereafter denoted FP) carried out detailed calculations of the equation of state of symmetric nuclear matter (SNM) containing equal numbers of protons and neutrons and of pure neutron matter (PNM) with VCS, using the Urbana $v_{14}$ model of the NN interaction [10]. In these calculations, effects of many-body forces were introduced partly via a small density dependence in the NN interaction and partly by adding a density-dependent contribution, attributed to the attractive two-pion exchange three-nucleon interaction [11]. The parameters of these contributions were adjusted to reproduce the empirical properties of SNM, and methods were developed to interpolate between SNM and PNM [12]. The main weakness in this approach is that the effect of many-body interactions on the structure of matter is ignored.

In the 1980s, a variational theory using Monte Carlo methods (VMC) was developed for light nuclei [13]. Correlation operators with the same structure were employed in both VMC and VCS methods, with the aim of obtaining a unified description of light nuclei and nuclear matter. The Urbana VII (UVII) model of three nucleon interactions, containing the Fujita-Miyazawa two-pion exchange three-nucleon interaction [14] and a phenomenological, shorter range interaction, was added to the Hamiltonian. The parameters of the UVII were 
determined by reproducing the binding energies of ${ }^{3} \mathrm{H},{ }^{4} \mathrm{He}$ and the equilibrium density of SNM using approximate VMC and VCS calculations [15].

Wiringa, Fiks and Fabrocini [16] (hereafter denoted WFF) calculated the equation of state of SNM and PNM with an improved VCS method using the Urbana and Argonne [17] $v_{14}$ models of the NN interaction and the Urbana VII three-nucleon interaction. Their calculations of PNM indicated a transition to a new phase, possibly including pion condensation, at $\rho \sim 0.24 \mathrm{fm}^{-3}$ with the Argonne $v_{14}$, though not with Urbana $v_{14}$. No evidence of a similar transition in SNM was found by WFF.

Since the pioneering work of Migdal [18] and of Sawyer and Scallapino [19], many investigators have used effective interactions to study the possibility of pion condensation in SNM and PNM. These efforts were recently reviewed by Kunihiro et al. [20]. In these studies, the $\Delta$-resonance is explicitly considered as a non-nucleonic degree of freedom. In contrast, WFF considered only nucleonic degrees of freedom and absorbed the effect of the $\Delta$-resonance into the two- and three-nucleon interactions. The approaches used by Migdal and by WFF to study pion condensation are thus very different. Although the effect of this type of transition on the equation of state is relatively small, it can have important consequences for the cooling and evolution of neutron stars [21].

In the late 1980s Carlson [22] developed the Green's function Monte Carlo (GFMC) method, with which exact calculations of light nuclei are possible, starting from Hamiltonians with realistic two- and three-nucleon interactions. The GFMC method can be used to determine some of the parameters of the three-nucleon interaction from exact calculations of nuclear binding energies.

Recently, Wiringa, Stoks and Schiavilla 23] made significant improvements to the Urbana-Argonne $v_{14}$ models of the $\mathrm{NN}$ interaction by including isospin symmetry breaking terms. Their resulting Argonne $v_{18}$ is one of the new models fit to the Nijmegen NN scattering database 24,25]. Using this interaction, along with the GFMC method, Pudliner et al. [26] obtained parameters for the Urbana IX (UIX) model of the three-nucleon interaction. Exact GFMC calculations by Pudliner et al. 27,28 have shown that the Argonne 
$v_{18}$ and Urbana IX interactions provide a good description of all bound states, up to seven nucleons. While all observed energies are not exactly reproduced, in each case the difference between theory and experiment is much smaller than the contribution of the three-nucleon interaction.

In the present work, we employ the Argonne $v_{18}$ and Urbana IX interactions to study the properties of SNM and PNM, using the VCS method. We have introduced further improvements to the method, in order to address the relatively strong momentum dependence of the Argonne $v_{18}$ interaction. These improvements are described in Appendices $\mathrm{A}$ and B.

With these interactions, we find that both SNM and PNM undergo transitions to a new phase at densities of $\sim 0.32 \mathrm{fm}^{-3}$ and $\sim 0.2 \mathrm{fm}^{-3}$, respectively. The transitions are similar in nature to that found by WFF in PNM, using the Argonne $v_{14}$ and Urbana model VII.

In this paper, we discuss the phase transition and the spin-isospin structure of normal SNM at equilibrium density. Additional results for the equation of state of dense matter, including relativistic effects, will be reported separately. The plan of the present paper is as follows. The Hamiltonian and the VCS method are reviewed in Sections II and III. In Section IV, we present various pair distribution functions of interest, at both equilibrium and transition densities. These results clearly indicate that in normal SNM at $\rho=0.16 \mathrm{fm}^{-3}$, the tensor correlations in $\mathrm{T}=0$ states have near maximum possible strength at $r \sim 1 \mathrm{fm}$. The results also indicate the important role played by the short-range parts of $\boldsymbol{\sigma}_{i} \cdot \boldsymbol{\sigma}_{j} \boldsymbol{\tau}_{i} \cdot \boldsymbol{\tau}_{j}$ and $\boldsymbol{\sigma}_{i} \cdot \boldsymbol{\sigma}_{j}$ interactions in determining the transition density, as predicted by Migdal. In Migdal's approach, the strength of the short-range part of the $\boldsymbol{\sigma}_{i} \cdot \boldsymbol{\sigma}_{j} \boldsymbol{\tau}_{i} \cdot \boldsymbol{\tau}_{j}$ interaction is represented by the Landau-Migdal parameter $g^{\prime}$. We also comment on the model dependence of this transition in Section IV, by comparing the Urbana $v_{14}$ and the Argonne $v_{14}$ and $v_{18}$ interactions. Results for the sums of isovector spin-longditudinal responses of SNM and PNM are presented in Section V. The corresponding results for the strengths of pion exchange interactions and pion fields appear in section VI. These results clearly indicate that the high density phase includes pion condensation, as anticipated by WFF. Results for SNM are presented at normal density and at the transition density. The response of SNM 
at normal density exhibits little indication of the phase transition, which is evidently of first order in the present approach. Conclusions are presented in Section VII.

\section{THE HAMILTONIAN}

The Argonne $v_{18}$ two-nucleon interaction has the form:

$$
v_{18, i j}=\sum_{p=1,18} v^{p}\left(r_{i j}\right) O_{i j}^{p}+v_{e m}
$$

The electromagnetic part $v_{e m}$ consists of Coulomb and magnetic interactions in the nn, np and pp pairs, and it is omitted from all nuclear matter studies. The strong interaction part of the potential includes fourteen isoscalar terms with operators:

$$
O_{i j}^{p=1,14}=\left[1, \boldsymbol{\sigma}_{i} \cdot \boldsymbol{\sigma}_{j}, S_{i j},(\mathbf{L} \cdot \mathbf{S})_{i j}, L^{2}, L^{2} \boldsymbol{\sigma}_{i} \cdot \boldsymbol{\sigma}_{j},(\mathbf{L} \cdot \mathbf{S})_{i j}^{2}\right] \otimes\left[1, \boldsymbol{\tau}_{i} \cdot \boldsymbol{\tau}_{j}\right]
$$

By convention, operators with even $p \leq 14$ include the $\boldsymbol{\tau}_{i} \cdot \boldsymbol{\tau}_{j}$ factor, while those with odd $p \leq 13$ are independent of isospin. The operators with $p=15,16$ and 17 are isotensor:

$$
O_{i j}^{p=15,17}=\left(3 \tau_{z i} \tau_{z j}-\boldsymbol{\tau}_{i} \cdot \boldsymbol{\tau}_{j}\right) \otimes\left(1, \boldsymbol{\sigma}_{i} \cdot \boldsymbol{\sigma}_{j}, S_{i j}\right)
$$

The interactions associated with operators $p=16$ and 17 are generated by the mass difference between neutral and charged pions, while the phenomenological interaction with the $p=15$ operator is necessary to fit the difference between $\mathrm{np}$ and $\mathrm{pp},{ }^{1} S_{0}$ phase shifts. Finally, the isovector interaction with the operator,

$$
O_{i j}^{18}=\left(\tau_{z i}+\tau_{z j}\right)
$$

is required to fit the nn scattering length and effective range along with the np and pp data. The interaction constructed with the above elements provides an accurate fit to the NN scattering data in the Nijmegen database, using the kinetic energy operator:

$$
K=\sum_{i}-\frac{\hbar^{2}}{4}\left[\left(\frac{1}{m_{p}}+\frac{1}{m_{n}}\right)+\left(\frac{1}{m_{p}}-\frac{1}{m_{n}}\right) \tau_{z i}\right] \nabla_{i}^{2},
$$

which takes into account the mass difference between protons and neutrons. 
The Urbana models of $V_{i j k}$ contain two isoscalar terms:

$$
V_{i j k}=V_{i j k}^{2 \pi}+V_{i j k}^{R} .
$$

The first term represents the Fujita-Miyazawa two-pion exchange interaction:

$$
\begin{gathered}
V_{i j k}^{2 \pi}=\sum_{c y c} A_{2 \pi}\left(\left\{\boldsymbol{\tau}_{i} \cdot \boldsymbol{\tau}_{j}, \boldsymbol{\tau}_{i} \cdot \boldsymbol{\tau}_{k}\right\}\left\{X_{i j}, X_{i k}\right\}+\frac{1}{4}\left[\boldsymbol{\tau}_{i} \cdot \boldsymbol{\tau}_{j}, \boldsymbol{\tau}_{i} \cdot \boldsymbol{\tau}_{k}\right]\left[X_{i j}, X_{i k}\right]\right), \\
X_{i j}=S_{i j} T_{\pi}\left(r_{i j}\right)+\boldsymbol{\sigma}_{i} \cdot \boldsymbol{\sigma}_{j} Y_{\pi}\left(r_{i j}\right),
\end{gathered}
$$

with strength $A_{2 \pi}$. The functions $T_{\pi}\left(r_{i j}\right)$ and $Y_{\pi}\left(r_{i j}\right)$ describe the radial shapes of the one-pion exchange tensor and Yukawa potentials. These functions are calculated using the average value of the pion mass and include the short-range cutoffs used in the Argonne $v_{18}$ $\mathrm{NN}$ interaction. The term denoted by $V_{i j k}^{R}$ is purely phenomenological, and has the form:

$$
V_{i j k}^{R}=U_{0} \sum_{c y c} T_{\pi}^{2}\left(r_{i j}\right) T_{\pi}^{2}\left(r_{i k}\right) .
$$

This term is meant to represent the modification of $\mathrm{N} \Delta$ - and $\Delta \Delta$-contributions in the twobody interaction by other particles in the medium, and also accounts for relativistic effects. The spin-isospin dependence of these effects is neglected.

The two parameters $A_{2 \pi}$ and $U_{0}$ are chosen to yield the observed energy of ${ }^{3} \mathrm{H}$ and the equilibrium density of nuclear matter, $\rho_{0}=0.16 \mathrm{fm}^{-3}$. The parameters of Model VII, used by WFF, were determined from approximate variational calculations using the older Argonne $v_{14} \mathrm{NN}$ interaction, and have the values: $A_{2 \pi}=-0.0333 \mathrm{MeV}$ and $U_{0}=0.0038$ $\mathrm{MeV}$. The parameters of Model IX, $A_{2 \pi}=-0.0293 \mathrm{MeV}$ and $U_{0}=0.0048 \mathrm{MeV}$, have been determined from exact GFMC calculations of ${ }^{3} \mathrm{H}$ and the present approximate variational calculations of nuclear matter with the Argonne $v_{18} \mathrm{NN}$ interaction. Most of the difference between the parameters of Models VII and IX is due to the use of exact, rather than approximate calculations of ${ }^{3} \mathrm{H}$ in the latter. The parameters of Model VIII, also determined via exact ${ }^{3} \mathrm{H}$ calculations, albeit with the older Argonne $v_{14}$ interaction, have the values: $A_{2 \pi}=-0.028 \mathrm{MeV}$ and $U_{0}=0.005 \mathrm{MeV}$, which are not very different from the present Model IX parameters. The insensitivity of these parameters to the details of the UrbanaArgonne models of $v_{i j}$ had been previously noted [29]. For example, calculations of ${ }^{3} \mathrm{H}$ and 
nuclear matter saturation density using Model VII yielded similar results with the Urbana $v_{14}$ and Argonne $v_{14}$ interactions, despite significant differences in the tensor components of these interactions. We also note that the values of $A_{2 \pi}$ obtained from all these fits are close to $\sim-0.03 \mathrm{MeV}$, the strength predicted by the Fujita-Miyazawa model. More recent theoretical models of $V_{i j k}^{2 \pi}$, such as the Tucson-Melbourne [30], predict significantly larger strengths, $A_{2 \pi} \sim-0.063 \mathrm{MeV}$. These models of $V_{i j k}^{2 \pi}$ also have additional terms which are neglected here.

The isotensor and isovector parts of $v_{i j}$, and the isovector part of the kinetic energy $\mathrm{K}$, are very weak and may presumably be treated as first order perturbations. In first order, these terms do not contribute to the energy of SNM, which has total isospin $\mathrm{T}=0$. Therefore, the SNM calculations, which neglect $v_{e m}$ by definition, are carried out with the isoscalar part of the Hamiltonian:

$$
H_{S N M}=\sum_{i}-\frac{\hbar^{2}}{4}\left(\frac{1}{m_{p}}+\frac{1}{m_{n}}\right) \nabla_{i}^{2}+\sum_{i<j} \sum_{p=1,14} v^{p}\left(r_{i j}\right) O_{i j}^{p}+\sum_{i<j<k} V_{i j k} .
$$

The isotensor and isovector terms do contribute to the energy of PNM. The isospin operators $\boldsymbol{\tau}_{i} \cdot \boldsymbol{\tau}_{j}, 3 \tau_{z i} \tau_{z j}-\boldsymbol{\tau}_{i} \cdot \boldsymbol{\tau}_{j}$ and $\tau_{z i}+\tau_{z j}$ reduce to constants, respectively equal to 1,2 and -2 , in PNM. Hence, the full Hamiltonian, excluding $v_{e m}$, has a simple form for PNM, given by:

$$
H_{P N M}=\sum_{i}-\frac{\hbar^{2}}{2 m_{n}} \nabla_{i}^{2}+\sum_{i<j} \sum_{q=1,7} \tilde{v}^{2 q-1}\left(r_{i j}\right) O_{i j}^{2 q-1}+\sum_{i<j<k} V_{i j k},
$$

with

$$
\begin{aligned}
\tilde{v}^{1} & =v^{1}+v^{2}+2 v^{15}-2 v^{18} \\
\tilde{v}^{3} & =v^{3}+v^{4}+2 v^{16} \\
\tilde{v}^{5} & =v^{5}+v^{6}+2 v^{17} \\
\tilde{v}^{2 q-1} & =v^{2 q-1}+v^{2 q} \text { for } q \geq 4
\end{aligned}
$$

We note that $\left[\boldsymbol{\tau}_{i} \cdot \boldsymbol{\tau}_{j}, \boldsymbol{\tau}_{i} \cdot \boldsymbol{\tau}_{k}\right]=0$ in PNM, thus the commutator term of $V_{i j k}^{2 \pi}$ can be omitted from $\mathrm{H}_{P N M}$. 


\section{VARIATIONAL CALCULATIONS}

The variational method developed in References [3, 11, 16, 31] provides a means to calculate the energy and wavefunction of the ground state of nuclear matter from realistic models of nuclear forces. We outline the method here and describe additional developments in Appendices A and B.

The variational wavefunction has the form:

$$
\Psi_{v}=\left(S \prod_{i<j} F_{i j}\right) \Phi
$$

consisting of a symmetrized product of pair correlation operators $F_{i j}$ operating on the Fermi gas wavefunction $\Phi$. In SNM, the $F_{i j}$ include eight terms:

$$
F_{i j}=\sum_{p=1,8} f^{p}\left(r_{i j}\right) O_{i j}^{p}
$$

representing central, $\boldsymbol{\sigma}_{i} \cdot \boldsymbol{\sigma}_{j}, \mathrm{~S}_{i j}$ and $(\mathbf{L} \cdot \mathbf{S})_{i j}$ correlations with and without $\boldsymbol{\tau}_{i} \cdot \boldsymbol{\tau}_{j}$ factors. In PNM, the $F_{i j}$ reduce to a sum of four terms with only odd $p \leq 7$.

This wavefunction is clearly too simple to accurately describe the ground state of nuclear matter. Monte Carlo studies of few-body nuclei [32] and ${ }^{16} \mathrm{O}$ [6] use additional three-body correlation operators in the variational wavefunction. These additions to the wavefunction lower the ground state energy of ${ }^{16} \mathrm{O}$ by $\sim 1 \mathrm{MeV} /$ nucleon. Attempts to include three-body correlation operators in variational calculations of SNM are currently in progress 33. Exact GFMC calculations have now been carried out for nuclei containing up to seven nucleons 26, 28. The ground state energy of ${ }^{7} \mathrm{Li}$ obtained with variational wavefunctions including three-body correlation operators is greater than the exact value by $\sim 0.7 \mathrm{MeV} /$ nucleon. From these results, we estimate that the present $\Psi_{v}$ may underbind SNM by a few MeV. In contrast, the three-body correlations have a much smaller effect on the energy of pure neutron drops [8]. The variational energy of a drop with eight neutrons, calculated with the simple $\Psi_{v}$, is greater than the exact value by $\sim 0.5 \mathrm{MeV} /$ nucleon. Thus, we believe the energies calculated in the present work to be relatively more accurate for PNM than for 
SNM. This result is to be expected, since SNM has strong tensor correlations in two-body np states, with isospin $\mathrm{T}=0$, and in three-body nnp and pnn clusters with $\mathrm{T}=\frac{1}{2}$.

Despite the aforementioned shortcomings, the simple $\Psi_{v}$ having only pair correlation operators describes the gross features of the nuclear wavefunction rather well. For example, the spin-isospin dependent two-nucleon distribution functions calculated in this approximation are close to the exact distribution functions [27]. We note that the magnitude of the ground state energy is generally much smaller than those of the positive kinetic and negative interaction energies. The magnitude of the error in the ground state energy is typically only a few percent of the interaction energy. We can therefore study the main features of the spin-isospin structure of PNM and SNM using the simple $\Psi_{v}$ described above.

The correlation operators $F_{i j}$ are determined from Euler-Lagrange equations [11] that minimize the two-body cluster contribution of an interaction $(\bar{v}-\lambda)$, where:

$$
\begin{aligned}
\bar{v}_{i j} & =\sum_{p=1,14} \alpha^{p} v^{p}\left(r_{i j}\right) O_{i j}^{p}, \\
\lambda_{i j} & =\sum_{p=1,8} \lambda^{p}\left(r_{i j}\right) O_{i j}^{p} .
\end{aligned}
$$

The variational parameters $\alpha^{p}$ are meant to simulate the quenching of the spin-isospin interaction between particles $i$ and $j$, due to flipping of the spin and/or isospin of particle $i$ or $\mathrm{j}$ via interaction with other particles in matter [34]. We use:

$$
\begin{aligned}
& \alpha^{p}=1 \text { for } p=1 \text { and } 9 \\
& \alpha^{p}=\alpha \text { otherwise }
\end{aligned}
$$

since the operators 1 and $L^{2}\left(p=1\right.$ and 9) are independent of spin-isospin. The $\alpha^{p}$ of the $(\mathbf{L} \cdot \mathbf{S})_{i j}^{2}$ interactions $(p=13,14)$ are also set to unity. The $(\mathbf{L} \cdot \mathbf{S})_{i j}^{2}$ operator has a significant $L^{2}$ part, which should not be quenched, and quenching the remainder of $v^{p=13}$, along with $v^{p=14}$, does not lower the variational energy. The $\lambda^{p}(r)$ simulate screening effects in matter and are determined by the healing distances $d^{p}$ of the correlation functions $f^{p}$ :

$$
f^{p}\left(r>d^{p}\right)=\delta_{p 1} .
$$


The radial dependence of $\lambda^{p}\left(r>d^{p}\right)$ is determined from the above constraint. The $\lambda^{p}\left(r<d^{p}\right)$ are constants chosen to make the gradients of $f^{p}$ at $d^{p}$ equal to zero. The energy of nuclear matter is particularly sensitive to the range of the tensor and central correlations. For simplicity we assume:

$$
\begin{aligned}
& d^{p}=d_{t} \text { for } p=5,6 \\
& d^{p}=d_{c} \text { for } p \neq 5,6 .
\end{aligned}
$$

The $F_{i j}$, and consequently $\Psi_{v}$, thus depend on three variational parameters: $\alpha, \mathrm{d}_{c}$ and $\mathrm{d}_{t}$, the values of which are determined by minimizing the energy. Two additional parameters used by WFF have only a small influence on the energy.

It is convenient to divide the interaction and correlation operators as follows:

$$
\begin{gathered}
v_{i j}=v_{s, i j}+v_{b, i j}+v_{q, i j}, \\
F_{i j}=F_{s, i j}+F_{b, i j} .
\end{gathered}
$$

The static parts $v_{s}$ and $F_{s}$ involve terms with momentum independent operators $O_{i j}^{p=1-6}$. The spin-orbit terms, with $p=7$ and 8 are included in $v_{b}$ and $F_{b}$, while terms quadratic in $L$, with $p=9$ to 14 are included in $v_{q}$. The two-body $(2 \mathrm{~B})$ cluster contributions involve expectation values of the operators:

$$
\begin{gathered}
f^{q}\left(r_{i j}\right) O_{i j}^{q} v_{i j}^{p} O_{i j}^{p} f^{q^{\prime}}\left(r_{i j}\right) O_{i j}^{q^{\prime}}, \\
f^{q}\left(r_{i j}\right) O_{i j}^{q} \nabla_{i j}^{2} f^{q^{\prime}}\left(r_{i j}\right) O_{i j}^{q^{\prime}},
\end{gathered}
$$

in plane-wave states. We divide these contributions into five parts: the static parts, $\langle v\rangle-2 \mathrm{~B}-\mathrm{s}$ $(\langle T\rangle-2 \mathrm{~B}-\mathrm{s})$ include interaction (kinetic) energy contributions for $q, p$ and $q^{\prime}\left(q\right.$ and $\left.q^{\prime}\right) \leq 6$; the spin-orbit parts, $\langle v\rangle-2 \mathrm{~B}-\mathrm{b}$ and $\langle T\rangle-2 \mathrm{~B}-\mathrm{b}$ include terms with $p \leq 8$, and one or more of the $p, q$, and $q^{\prime}$ equal to 7 or 8 ; and the quadratic part, $\langle v\rangle-2 \mathrm{~B}$-q includes all terms with $p \geq$ 9 .

The results obtained with various Hamiltonians for SNM at $\mathrm{k}_{f}=1.33 \mathrm{fm}^{-3}$ and $1.6 \mathrm{fm}^{-3}$

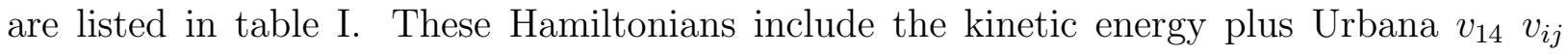


(U14), Argonne $v_{18} v_{i j}(\mathrm{~A} 18)$ and Argonne $v_{18} v_{i j}$ plus Model IX $V_{i j k}$ (A18+IX). The Fermi kinetic energy is listed as $\langle T\rangle-1 \mathrm{~B}$, the one-body cluster contribution to the kinetic energy.

Variational cluster Monte Carlo studies of ${ }^{8} \mathrm{n}$-drops [8] and ${ }^{16} \mathrm{O}$ [6, 35], including up to five-body cluster contributions to the ground state energy, indicate that the convergence of the cluster expansion is not particularly good in either case. For example, the onethrough five-body clusters contribute $12.9,-54.5,11.1,-3.8$, and $1.1 \mathrm{MeV}$ respectively to the ground state energy of the ${ }^{8} \mathrm{n}$-drop. This result does not contradict the earlier lowestorder constrained variational calculations of neutron gas [36,37], which retained only the $\langle T\rangle-1 \mathrm{~B}$ and all $2 \mathrm{~B}$ contributions. The aim of the earlier studies was to obtain estimates of the ground state energy; the $d_{c}$ and $d_{t}$ were not determined variationally, but rather were fixed by constraints. In the present work, $\Psi_{v}$ is determined variationally to study the structure of SNM and PNM. The larger optimum values of $d_{c}$ and $d_{t}$ lead to the significant size of many-body (MB) clusters of three or more particles.

According to Table $\mathbb{q}$, the kinetic energy and static interactions and correlations make large contributions to the $1 \mathrm{~B}$ and $2 \mathrm{~B}$ cluster energies. We expect MB cluster contributions containing $\nabla_{i}^{2}$ or $v_{s, i j}$ and $F_{s}$ correlations to be important. In this work, as in earlier studies by FP and WFF, these contributions are calculated using chain summation methods [3]. The following three improvements were made by WFF in the basic VCS calculation used by FP:

(i) The kinetic energy can be calculated using different expressions related by integration by parts. If all $\mathrm{MB}$ contributions are calculated, these expressions yield the same result. However, they yield different results when only selected parts of the MB clusters are summed by VCS techniques. Studies of atomic helium liquids with VCS methods [38] find the exact result to be between the energies obtained using the Jackson-Feenberg (JF) and Pandharipande-Bethe (PB) expressions. Both the JF and PB energies were calculated by WFF, who took the average of the two as the result, and half the difference as an estimate of the error.

(ii) The pair distribution function $g^{c}(r)$ is defined such that $\rho g^{c}(r)$ represents the prob- 
ability of finding a particle at a distance $\mathrm{r}$ from a given particle. Conservation of number of particles then implies:

$$
I_{c}=\rho \int d^{3} r\left(1-g^{c}(r)\right)=1
$$

for both SNM and PNM. Also, since SNM has total isospin T=0, we have the following identity for expectation values of $\boldsymbol{\tau}_{i} \cdot \boldsymbol{\tau}_{j}$ operators:

$$
I_{\tau}=\frac{1}{A}\left\langle 0\left|\sum_{i, j=1, A} \boldsymbol{\tau}_{i} \cdot \boldsymbol{\tau}_{j}\right| 0\right\rangle=-3 .
$$

The values of $\mathrm{I}_{c}$ and $\mathrm{I}_{\tau}$ calculated using the VCS method are generally within a few percent of these exact results. However at small densities, the correlations become large due to the bound deuteron and virtual bound ${ }^{1} \mathrm{~S}_{0}$ states, causing $\mathrm{I}_{c}$ and $\mathrm{I}_{\tau}$ to deviate from their exact values by more than $10 \%$ in some regions of the $d_{c}, d_{t}, \alpha$ parameter space. Deviations of this size can also occur at large densities. Excursions into such regions of the parameter space are curtailed in SNM by minimizing:

$$
\langle H\rangle+\Lambda\left[\left(I_{c}-1\right)^{2}+\left(\frac{1}{3} I_{\tau}+1\right)^{2}\right]
$$

with $\Lambda$ chosen to keep $I_{c}$ and $I_{\tau}$ within $10 \%$ of their exact values. Only the $\Lambda\left(I_{c}-1\right)^{2}$ constraint is applicable for PNM.

(iii) In their calculation, WFF added the leading multiple-operator chain contributions to those summed via VCS. At $\rho_{0}$, these terms contribute less than $1 \mathrm{MeV} /$ nucleon, while at $4 \rho_{0}$, they may contribute a few $\mathrm{MeV} /$ nucleon. Including these terms, WFF estimate the error in the calculation of the MB contributions due to kinetic energy, $v_{s}$ and $F_{s}$ to be $\sim$ $0.5 \mathrm{MeV} /$ nucleon at $\rho_{0}$, and $\sim 5 \mathrm{MeV} /$ nucleon at $6 \rho_{0}$ in $\mathrm{SNM}$; the corresponding error in PNM is lower still. We note that these errors are negligible compared to the error implicit in the use of the simple $\Psi_{v}$ given by Eq. (3.1).

The computer program used to carry out the present calculations is built upon that of WFF and retains all the improvements made by them.

The contribution of $\mathrm{MB}$ clusters involving spin-orbit correlations and interactions is calculated using methods developed by Lagaris [31. Separable three-body clusters, with 
correlations between pairs ij and ik, but not between $\mathrm{jk}$, make the dominant contribution to $\langle v+T\rangle$-MB-b, via $v_{i j}$ or $\nabla_{i}^{2}$ terms in the Hamiltonian. Chain diagrams with correlations between all three pairs ij,ik and jk were found to make a smaller contribution. We therefore sum all three-body separable contributions to $\langle v+T\rangle$-MB-b, and estimate only the leading chain contributions.

Lagaris also calculated part of the three-body separable contribution to $\langle v\rangle$-MB-q using the U14 Hamiltonian [11]. The $L^{2}$ and $(\mathbf{L} \cdot \mathbf{S})^{2}$ interactions that contribute to $\langle v\rangle$-MB-q are significantly stronger in the A18 model than in the U14 model. We therefore include a more complete calculation of three-body separable terms and leading central chain contributions to $\langle v\rangle$-MB-q in the present work. These calculations are outlined in appendix A. The present calculation of $\langle v\rangle$-MB-q and the earlier calculation by Lagaris yield similar results for the U14 Hamiltonian: 1.61 (3.91) $\mathrm{MeV}$ versus 1.35 (3.0) $\mathrm{MeV}$ at $k_{F}=1.33$ (1.6) $\mathrm{fm}^{-3}$. The difference between the the two calculations is significant for the the A18+IX Hamiltonian,

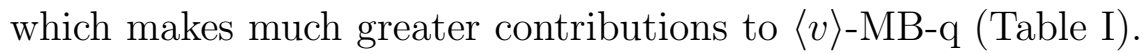

We have summed the contributions of leading three-body and more than three-body diagrams to the expectation values of the $V^{2 \pi}$ and $V^{R}$ static three-body interactions, using VCS methods described in Reference [39]. The A18 interaction induces stronger correlations than U14, as is evident by comparing T-2B-s for the two interactions in Table [I. The larger correlations lead to comparatively larger $\left\langle V^{2 \pi}\right\rangle$ and $\left\langle V^{R}\right\rangle$. The $\left\langle V^{2 \pi}\right\rangle$ in U14+VII [15] and A18+IX models equal -3.49 and $-3.60 \mathrm{MeV}$ respectively at $k_{F}=1.33 \mathrm{fm}^{-3}$, despite the smaller strength of $V^{2 \pi}$ in Model IX $\left(A_{2 \pi}=-0.0293 \mathrm{MeV}\right)$ compared to Model VII $\left(A_{2 \pi}=\right.$ $-0.0333 \mathrm{MeV})$. The $\left\langle V^{R}\right\rangle$ is larger for $\mathrm{A} 18+\mathrm{IX}\left(6.33 \mathrm{MeV}\right.$ at $\left.k_{F}=1.33 \mathrm{fm}^{-3}\right)$ compared to $\mathrm{U} 14+\mathrm{VII}\left(3.99 \mathrm{MeV}\right.$ at $\left.k_{F}=1.33 \mathrm{fm}^{-3}\right)$. Only about half the increase in $\left\langle V^{R}\right\rangle$ is due to the larger strength of $V^{R}$ in Model IX $\left(U_{0}=0.0048 \mathrm{MeV}\right)$ compared to Model VII $\left(U_{0}=0.0038\right.$ $\mathrm{MeV})$.

We note that the two-body correlations in this variational calculation do not have the optimal form. The $F_{i j}$ are obtained by minimizing the sum of $2 \mathrm{~B}$ contributions of the potential $\bar{v}$ (Eq. 3.3), with healing constraints imposed at $d_{c}$ and $d_{t}$. More general correlations can be 
generated by separately minimizing the two-body cluster contribution to each partial wave, specified by $l, S, J$ and the relative momentum $k$ 40. Thus, the correlations $f(l, S, J, k)$ depend on all the quantum numbers, and yield a lower $2 \mathrm{~B}$ energy than the $F_{i j}$ operators defined in Eq. (3.2) for $\bar{v}$ with the same $d_{c}$ and $d_{t}$. The MB contributions cannot be easily calculated with the general $f(l, S, J, k)$, however.

The $F_{i j}$ operators provide a good approximation to the two-body correlations in matter. The small differences between optimum $f(l, S, J, k)$ and $F_{i j}$ can be accounted for by inclusion of the second order two-particle, two-hole contribution, $\Delta E_{2}$, calculated in correlated basis perturbation theory 44, 42. We estimate this contribution as described in Appendix B, approximating the $\Delta E_{2}$ by the difference $\delta E_{2 B}$ between the $2 \mathrm{~B}$ cluster energies calculated using $f(l, S, J, k)$ and $F_{i j}$. The values of $\alpha, d_{c}, d_{t}$ are determined by minimizing the energy calculated from the $F_{i j}$, and $\delta E_{2 B}$, calculated for these optimum $\alpha, d_{c}, d_{t}$, is perturbatively

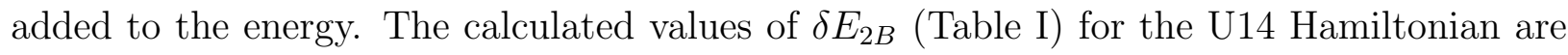
slightly smaller in magnitude than the $\Delta E_{2}$ values reported in Reference [41], for the same interaction. The $\delta E_{2 B}$ correction is larger for the A18 and A18+IX Hamiltonians, which predict stronger correlations in matter. The $\Delta E_{2}$ is known to be relatively larger for the A14 interaction [42], which induces stronger correlations than the U14. Our best estimate of the variational energy, given by $\frac{1}{2}\left(E_{P B}+E_{J F}\right)+\delta E_{2 B}$, is also listed in Table \.

Over a decade ago, Day and Wiringa [5] calculated the ground state energies of SNM by means of the Brueckner-Bethe method. Contributions of up to four hole-line diagrams were included, in order to reduce the estimated error in the calculated energy to $\sim \pm 0.18(1.3) \mathrm{MeV}$ at $k_{f}=1.33(1.6) \mathrm{fm}^{-1}$. The calculations demonstrated that the three realistic, two-body potentials used in that work, Bonn (1975) 44,44, Paris 45, and A14, yielded essentially the same energy of $\sim-14.9 \mathrm{MeV}$ at $k_{f}=1.33 \mathrm{fm}^{-1}$. At $k_{f}=1.6 \mathrm{fm}^{-1}$, the Paris and Argonne $v_{14}$ models yielded similar energies of $\sim-17.8 \mathrm{MeV}$, while the Bonn result was somewhat higher, at $\sim-16.8 \mathrm{MeV}$. Our results with U14 and A18 (Table II) are close to the earlier results at $k_{f}=1.33 \mathrm{fm}^{-1}$, and are about $1 \mathrm{MeV}$ lower at $k_{f}=1.6 \mathrm{fm}^{-1}$. As with the results of Day and Wiringa, the present results show a remarkable model independence 
of the energy of SNM for $k_{f} \leq 1.6 \mathrm{fm}^{-1}$, calculated from realistic two-nucleon interaction models. The differences in the energies obtained using the different models are smaller than the estimated error in the many-body calculations.

The energies of SNM and PNM calculated using the A18+IX Hamiltonian appear in Fig. 1 and Fig. 2, along with the results obtained by WFF using A14+VII. As discussed in detail below, there appears to be a phase transition in both SNM and PNM with the present Hamiltonian. The curves marked LDP and HDP show the energies obtained for the low and high densitiy phases, respectively.

At larger densities, the A18+IX energies are significantly higher than the A14+VII energies. A major part of this difference can be attributed to the difference between $V_{i j k}$ in Models VII and IX. For example, the contribution of Model IX exceeds that of Model VII by $8.2(4.5) \mathrm{MeV}$ to SNM (PNM) energy at $\rho=0.32 \mathrm{fm}^{-3}$. The Model VII is unrealistic, however, as it overbinds ${ }^{3} \mathrm{H}$ and ${ }^{4} \mathrm{He}$. The remainder of the difference between $\mathrm{A} 18+\mathrm{IX}$ and $\mathrm{A} 14+\mathrm{VII}$ is due to the stronger momentum-dependent part, $v_{q, i j}$ of A18, which makes significant contributions at larger densities.

In the early 1980s, FP added phenomenological density-dependent terms to the U14 Hamiltonian, and adjusted their parameters to reproduce the empirical equilibrium density, energy and compressibility of SNM. These results also appear in Fig. 1 and Fig. 2. Energy density functionals based on the FP results for $\mathrm{E}(\rho)$ of SNM and PNM reproduce the binding energies of nuclei from ${ }^{16} \mathrm{O}$ to ${ }^{208} \mathrm{~Pb}$ rather well [46].

The minimum energy calculated for SNM with the present variational wavefunction and the A18+IX Hamiltonian is $-12 \mathrm{MeV}$, compared to the empirical value of $-16 \mathrm{MeV}$. As previously mentioned, results of variational and exact calculations of light nuclei suggest that including three-body correlations in the wavefunction could lower the variational bound on the energy by more than $1 \mathrm{MeV}$, and that the true energy may be more than $1 \mathrm{MeV}$ lower still. Thus, the underbinding of SNM due solely to deficiencies in the A18+IX model of nuclear forces is probably much less than $2 \mathrm{MeV}$. This underbinding is a very small fraction of the total potential energy of SNM at $\rho_{0}=0.16 \mathrm{fm}^{-3}$, which is $\sim-50 \mathrm{MeV}$ for this 
Hamiltonian.

At approximately twice the equilibrium density, we observe what appears to be a transition in SNM due to a change in $d_{t}$, the range of tensor correlations. The energy of SNM is shown in Fig. 3 as a function of density and of $d_{t} / r_{0}$, where $r_{0}$ is the unit radius defined by

$$
\frac{4 \pi}{3} r_{0}^{3} \rho=1
$$

The energies in Fig. 3 have been minimized with respect to variations in the other two parameters, $\alpha$ and $d_{c}$, at each $\rho$ and $d_{t} / r_{0}$. For $\rho<0.32 \mathrm{fm}^{-3}$, the minimum occurs at $d_{t} / r_{0} \sim 4$, whereas for $\rho>0.32 \mathrm{fm}^{-3}$, it shifts to $d_{t} / r_{0} \sim 6$. The upper line in Fig. 1 1 , labeled LDP represents the minimum energies for $d_{t} / r_{0} \sim 4$, while the lower line labeled HDP gives the energies of the minima at larger values of $d_{t} / r_{0}$.

This transition is probably related to pion condensation [16, 18, 36], and its spin-isospin structure is discussed in the following sections. The transition does not occur in similar calculations of SNM using either the U14 or A14 and is thus sensitive to the two-nucleon interaction model. The Fujita-Miyazawa two-pion exchange three-nucleon interaction is a necessary ingredient for the transition to occur in SNM. A similar transition occurs for PNM (Fig. 2) at a lower density $\left(\sim 0.2 \mathrm{fm}^{-3}\right)$ with both the A14+VII and A18+IX Hamiltonians, but it is not observed for U14+VII. The $E\left(\rho, d_{t} / r_{0}\right)$ for PNM with A18+UIX appear in Fig. 4. Unlike the A14 and U14 interactions, the transition persists in PNM with A18, in the absence of the Fujita-Miyazawa interaction, although it occurs at a much higher density $\left(\sim 3.5 \rho_{0}\right)$ in that case.

The contributions to $E\left(\rho, d_{t} / r_{0}\right)$ of SNM at selected values of $\rho$ and $d_{t} / r_{0}$ are listed in Table $[1]$ for the A18+IX model. The interactions $v^{t \tau}, v^{\sigma \tau}$ and $v^{c}$, associated with the operators $S_{i j} \boldsymbol{\tau}_{i} \cdot \boldsymbol{\tau}_{j}, \boldsymbol{\sigma}_{i} \cdot \boldsymbol{\sigma}_{j} \boldsymbol{\tau}_{i} \cdot \boldsymbol{\tau}_{j}$ and 1, make the largest contributions to the energy of SNM. These contributions are listed separately, along with the contribution of the entire $v$. The $v^{t \tau}$ and $v^{\sigma \tau}$ contributions come mainly from the one-pion exchange interaction. The expectation values of $v^{\sigma \tau}, V^{2 \pi}$ and many-body cluster contributions are significantly different in the LDP and HDP. The pion-exchange contributions to the energies of PNM and SNM are listed in 
Table III for the LDP and HDP. The OPEP used to calculate $\left\langle v^{\pi}\right\rangle$ includes a $\pi N N$ dipole form factor with a $5 \mathrm{fm}^{-1}$ cutoff as described in Section VI. The $V^{2 \pi}$ is that given by Model IX. The results indicate that for SNM the change in the pion-exchange contribution between the two phases comes overwhelmingly from the $V^{2 \pi}$, which is required to produce the phase transition. However, for PNM a significant part of the enhancement in the pion-exchange contribution comes from $v^{\pi}$, thus indicating the diminished importance of the three-body interaction to the phase transition, and the persistence of the transition in the absence of $V^{2 \pi}$

In the present calculation, the transition in SNM is of first order, as is evident in Fig. 1. Thus, it is difficult to obtain information about the HDP by studying the LDP. The order of the corresponding transition in PNM is not as evident in Fig. 2, though it appears to be of first order as well.

\section{PAIR DISTRIBUTION FUNCTIONS}

The two-body density $\rho_{2}^{p}(r)$, associated with the operator $\mathrm{O}_{i j}^{p}$, is defined [6] such that:

$$
\left\langle 0\left|\sum_{i \neq j=1, A} B\left(r_{i j}\right) O_{i j}^{p}\right| 0\right\rangle=A \int d^{3} r B(r) \rho_{2}^{p}(r),
$$

for any function $\mathrm{B}\left(\mathrm{r}_{i j}\right)$. This relationship is used to calculate the expectation values of the pair interactions $v^{p}\left(r_{i j}\right) O_{i j}^{p}$ in the ground states of nuclei and nuclear matter, denoted by $|0\rangle$. The $\rho_{2}^{p}$ associated with the static operators $1, \boldsymbol{\tau}_{i} \cdot \boldsymbol{\tau}_{j}, \boldsymbol{\sigma}_{i} \cdot \boldsymbol{\sigma}_{j}, \boldsymbol{\sigma}_{i} \cdot \boldsymbol{\sigma}_{j} \boldsymbol{\tau}_{i} \cdot \boldsymbol{\tau}_{j}, S_{i j}$ and $S_{i j} \boldsymbol{\tau}_{i} \cdot \boldsymbol{\tau}_{j}$, are denoted by $\rho_{2}^{c}, \rho_{2}^{\tau}, \rho_{2}^{\sigma}, \rho_{2}^{\sigma \tau}, \rho_{2}^{t}$ and $\rho_{2}^{t \tau}$. All properties of SNM discussed in this and the following two sections are obtained from these six two-body densities. In the case of PNM, we disregard isospin and consider only $\rho_{2}^{c}, \rho_{2}^{\sigma}$ and $\rho_{2}^{t}$. The central two-body density, $\rho_{2}^{c}$, is proportional to the probability of finding a pair of particles separated by a distance $\mathrm{r}$, and asymptotically approaches the matter density, $\rho$, as $\mathrm{r} \rightarrow \infty$. All other $\rho_{2}^{p}$ are associated with spin-isospin correlations and must therefore vanish as $\mathrm{r} \rightarrow \infty$.

The static $\rho_{2}^{p}$, calculated for SNM at the equilibrium density of $\rho=0.16 \mathrm{fm}^{-3}$, appear in

Fig. 5. The large magnitudes of the $\rho_{2}^{p>1}$ indicate that short-range correlations in SNM are 
strongly spin-isospin dependent. The nature of these correlations is more evident in the pair distribution functions $\rho_{T, S, M}^{(2)}(\mathbf{r})$, which are proportional to the probability of finding a pair of nucleons with total isospin $\mathrm{T}$, spin $\mathrm{S}$ and spin projection $\mathrm{M}$, as a function of $\mathbf{r}$. Forest et al. recently studied these densities in light nuclei [7].

The $\rho_{T, 0,0}^{(2)}(r)$, in $\mathrm{S}=0, \mathrm{M}=0$ states, are spherically symmetric and are obtained from the $\rho_{2}^{p}$ 's using spin-isospin projection operators, such as $P_{S=0}=\frac{1}{4}\left(1-\boldsymbol{\sigma}_{i} \cdot \boldsymbol{\sigma}_{j}\right)$. In SNM,

$$
\begin{aligned}
& \rho_{1,0,0}^{(2)}(r)=\frac{1}{16}\left[3 \rho_{2}^{c}(r)+\rho_{2}^{\tau}(r)-3 \rho_{2}^{\sigma}(r)-\rho_{2}^{\sigma \tau}(r)\right], \\
& \rho_{0,0,0}^{(2)}(r)=\frac{1}{16}\left[\rho_{2}^{c}(r)-\rho_{2}^{\tau}(r)-\rho_{2}^{\sigma}(r)+\rho_{2}^{\sigma \tau}(r)\right] .
\end{aligned}
$$

Whereas in PNM,

$$
\rho_{0,0}^{(2)}(r)=\frac{1}{4}\left[\rho_{2}^{c}(r)-\rho_{2}^{\sigma}(r)\right]
$$

The pair distribution functions in $\mathrm{S}=1$ states have a quadropolar deformation due to the tensor interaction. The $\rho_{S, M}^{(2)}$ in PNM are given by:

$$
\begin{aligned}
\rho_{1,0}^{(2)}(\mathbf{r}) & =C_{0}(r)-2 C_{2}(r) P_{2}(\cos \theta) \\
\rho_{1, \pm 1}^{(2)}(\mathbf{r}) & =C_{0}(r)+C_{2}(r) P_{2}(\cos \theta)
\end{aligned}
$$

The expectation value of $B_{1}\left(r_{i j}\right) P_{S=1}$, where $P_{S=1}=\frac{1}{4}\left(3+\boldsymbol{\sigma}_{i} \cdot \boldsymbol{\sigma}_{j}\right)$, can be calculated from either the two-body densities $\rho_{2}^{(p)}$ or from the distributions $\rho_{1, M}^{(2)}$. Equating the two results, we find:

$$
C_{0}(r)=\frac{1}{3} \times \frac{1}{4}\left[3 \rho_{2}^{c}(r)+\rho_{2}^{\sigma}(r)\right]
$$

A similar calculation of the expectation value of $B_{t} S_{i j}$ yields:

$$
C_{2}(r)=\frac{1}{12} \rho_{2}^{t}(r)
$$

In the case of SNM, the $C_{0}(r)$ and $C_{2}(r)$ in $\mathrm{T}=0,1$ states are obtained from the expectation values of $B_{1}\left(r_{i j}\right) P_{S=1}$ and $B_{t} S_{i j}$, multiplied by isospin-projection operators, $P_{T=0,1}$. For the $\mathrm{T}=0, \mathrm{~S}=1$ distribution functions, we obtain: 


$$
\begin{aligned}
C_{0}(r) & =\frac{1}{3} \times \frac{1}{16}\left[3 \rho_{2}^{c}(r)-3 \rho_{2}^{\tau}(r)+\rho_{2}^{\sigma}(r)-\rho_{2}^{\sigma \tau}(r)\right], \\
C_{2}(r) & =\frac{1}{12} \times \frac{1}{4}\left[\rho_{2}^{t}(r)-\rho_{2}^{t \tau}(r)\right] .
\end{aligned}
$$

The corresponding $\mathrm{T}=1$ expressions are given by:

$$
\begin{aligned}
& C_{0}(r)=\frac{1}{3} \times \frac{1}{16}\left[9 \rho_{2}^{c}(r)+3 \rho_{2}^{\tau}(r)+3 \rho_{2}^{\sigma}(r)+\rho_{2}^{\sigma \tau}(r)\right] \\
& C_{2}(r)=\frac{1}{3} \times \frac{1}{16}\left[3 \rho_{2}^{t}(r)+\rho_{2}^{t \tau}(r)\right] .
\end{aligned}
$$

The extrema of $\rho_{T, 1, M}^{(2)}(r, \theta)$ at fixed $r$ occur for $M=0$ at $\theta=0$ and $\theta=\frac{\pi}{2}$. These extrema are plotted as a function of $r$ in Figs. 6,8 and 9. From Eqs. 4.5,4.6, it follows that:

$$
\begin{aligned}
\rho_{T, 1, \pm 1}^{(2)}(r, \theta=0) & =\rho_{T, 1,0}^{(2)}(r, \theta=\pi / 2), \\
\rho_{T, 1, \pm 1}^{(2)}(r, \theta=\pi / 2) & =\frac{1}{2}\left(\rho_{T, 1,0}^{(2)}(r, \theta=0)+\rho_{T, 1,0}^{(2)}(r, \theta=\pi / 2)\right) .
\end{aligned}
$$

We also note that in SNM:

$$
\rho_{T, S, M}^{(2)}(r \rightarrow \infty)=\frac{2 T+1}{16} \rho
$$

The $\rho_{0,1,0}^{(2)}(r, \theta=0, \pi / 2)$ are shown in Fig. 6 for SNM at $\rho=0.16 \mathrm{fm}^{-3}$, along with the corresponding functions for ${ }^{2} \mathrm{H},{ }^{4} \mathrm{He}$ and ${ }^{16} \mathrm{O}$, from Ref. [7]. At small $r$, this density is large for $\theta=\pi / 2$, where the OPE tensor potential is attractive, and small for $\theta=0$, where the OPEP is repulsive. Thus, according to Ref. [7], equidensity surfaces having $\rho_{0,1,0}^{(2)}>0.01$ $\mathrm{fm}^{-3}$ are toroidal in shape. The ratio $\rho_{0,1,0}^{(2)}(r, \theta=\pi / 2) / \rho_{0,1,0}^{(2)}(r, \theta=0)$ is a measure of the strength of tensor correlations in $\mathrm{T}=0$ states. For the maximum possible tensor correlations, $\rho_{0,1,0}^{(2)}(r, \theta=0)$ is negligible compared to $\rho_{0,1,0}^{(2)}(r, \theta=\pi / 2)$. Fig. 6 thus indicates that the tensor correlations in $\mathrm{T}=0$ states, in nuclei and in nuclear matter, have near the maximum possible strength at $r \sim 1 \mathrm{fm}$. The peak value of $\rho_{0,1, M}^{(2)}(\mathbf{r})$ is almost 2.5 times the asymptotic value of $0.01 \mathrm{fm}^{-3}$ at $\rho=0.16 \mathrm{fm}^{-3}$. The spherically symmetric two-body densities for SNM and nuclei in the $\mathrm{T}=1, \mathrm{~S}=0$ channel $\left(\rho_{1,0,0}^{(2)}(\mathbf{r})\right)$ are shown in Fig. 7. These distributions peak at $r \sim 1$, where the nuclear force is most attractive, and the peak value is about 1.5 times the asymptotic value. Both $\rho_{1,0,0}^{(2)}(\mathbf{r})$ and $\rho_{0,1, M}^{(2)}(\mathbf{r})$ are supressed near $r \sim 0$ by the repulsive core in the NN interaction. 
According to Ref. [7], the $\rho_{0,1, M}^{(2)}(\mathbf{r})$ and $\rho_{1,0,0}^{(2)}(\mathbf{r})$ have universal shapes in light nuclei at small $r$. In Figs. 6 and 7 , we have scaled the densities in light nuclei such that their maximum values equal those of SNM. The two-body density distributions in SNM appear to have nearly the same shape as those in light nuclei for $r \lesssim 1.5 \mathrm{fm}$. However, significant differences occur for $r \gtrsim 2 \mathrm{fm}$. Note that the average interparticle distance in SNM at $\rho=0.16 \mathrm{fm}^{-3}$ is also $\sim 2 \mathrm{fm}$.

Forest et al. argue in Ref. [7], that the ratio, $R_{A d}$, of the maximum values of $\rho_{0,1, M}^{(2)}$ in a nucleus $\mathrm{A}$ and the deuteron provides a good approximation to the Bethe-Levinger factor, $L_{A}$, of the nucleus $\mathrm{A}$. The pion and photon absorbtion cross sections in light nuclei scale with $R_{A d}$. The calculated value of $R_{A d}$ for SNM at equilibrium density is $1.59 \mathrm{~A}$, which corresponds to $L_{A}=6.36$.

We observe interesting changes in the two-body densities between the low- and highdensity phases in SNM and PNM. The existence of a pion condensate is indicated in the HDP, as discussed in the following sections. The $\rho_{T, S, M}^{(2)}(\mathbf{r})$ in SNM at $\rho=0.36 \mathrm{fm}^{-3}$ in the LDP, with $d_{t} / r_{0}=4$, and in the HDP, with $d_{t} / r_{0}=6$ are shown in Fig. 8. The analogous $\rho_{S, M}^{(2)}(\mathbf{r})$ in PNM at $\rho=0.20 \mathrm{fm}^{-3}$ are shown in Fig. 9. The differences between pair densities in LDP and HDP are more pronounced in PNM. In both PNM and SNM, we find that the long-range part of the tensor correlations is enhanced. In PNM, the $S=0 \rho_{0,0}^{(2)}$ is suppressed in the HDP, whereas in SNM, $\rho_{1,0,0}^{(2)}$ and $\rho_{0,1, M}^{(2)}$ are suppressed and $\rho_{0,0,0}^{(2)}$ is enhanced.

In Migdal's approach [18], the transition to the pion condensed phase is inhibited by a positive, short-range $\boldsymbol{\sigma}_{i} \cdot \boldsymbol{\sigma}_{j} \boldsymbol{\tau}_{i} \cdot \boldsymbol{\tau}_{j}$ two-nucleon interaction $v^{\sigma \tau}(r)$, represented by the Landau parameter $g^{\prime}$. The eigenvalues of $\boldsymbol{\sigma}_{i} \cdot \boldsymbol{\sigma}_{j} \boldsymbol{\tau}_{i} \cdot \boldsymbol{\tau}_{j}$ are $-3,-3,1$ and 9 in $\mathrm{T}, \mathrm{S}=1,0 ; 0,1 ; 1,1$; and 0,0 states, respectively. Therefore, a strong positive $v^{\sigma \tau}(r)$ favors the LDP, which has larger pair densities in $\mathrm{T}, \mathrm{S}=1,0$ and 0,1 states, and a smaller pair density in $\mathrm{T}, \mathrm{S}=0,0$. Similarly, in PNM, a large positive $\tilde{v}^{\sigma}$, given by Eq. 2.13, favors the LDP. The $v^{\sigma \tau}(r)$ and $\tilde{v}^{\sigma}(r)$ in the U14, A14 and A18 models are shown in Fig. 10. The positive $v^{\sigma \tau}$ and $\tilde{v}^{\sigma}$ of U14 prevent a transition to the HDP in both SNM and PNM. In A14, $\tilde{v}^{\sigma}$ becomes negative at small $r$, 
and PNM is thus predicted by WFF to undergo a transition, while in A18, both $v^{\sigma \tau}$ and $\tilde{v}^{\sigma}$ change sign and thus cannot prevent the transition in either SNM or PNM. The UrbanaArgonne potentials have similar forms, but are fit to different data sets. Whereas the U14 and A14 were fit to n-p phase shifts available in the late 1970s and early 1980s, respectively, the A18 is fit directly to the 1994 Nijmegen p-p and n-p scattering database. Also, a much better fit was achieved by A18 $\left(\chi^{2}\right.$ per datum $\left.=1.09\right)$. Thus, it is likely that A18 provides a more accurate representation of the NN interaction within the Urbana-Argonne framework.

The $v^{\sigma \tau}(r)$ predicted by $\pi$ - and $\rho$-exchange potentials is positive for point-like nucleons. However, these meson-exchange potentials also contain a negative $\delta$-function term. It is possible that this term is broadened by the finite size of nucleons, and that the total $v^{\sigma \tau}(r)$ changes sign at small $r$. The one-meson exchange representation of the NN interaction may not be reliable at small $r$, however.

The $v^{t \tau}(r) S_{i j} \boldsymbol{\tau}_{i} \cdot \boldsymbol{\tau}_{j}$ interaction contains the main part of the OPEP. At $r>1 \mathrm{fm}$ this interaction is essentially identical in the U14, A14 and A18 models. At $r<1 \mathrm{fm}$ it is strongest in A14 and weakest in U14.

\section{ISOVECTOR SPIN-LONGITUDINAL RESPONSE}

Migdal [18] calculated the isovector spin-longitudinal (IVSL) response of nuclear matter using effective interactions. The IVSL response is defined as:

$$
\begin{aligned}
R_{L}(q, \omega) & =\sum_{I}\left|\left\langle I\left|O_{L}(\mathbf{q})\right| 0\right\rangle\right|^{2} \delta\left(\omega_{I}-\omega_{0}-\omega\right), \\
O_{L}(\mathbf{q}) & =\sum_{i=1, A} \boldsymbol{\sigma}_{\mathbf{i}} \cdot \mathbf{q} \boldsymbol{\tau}_{\mathbf{i}} \cdot \hat{\mathbf{t}} e^{i \mathbf{q} \cdot \mathbf{r}_{\mathbf{i}}}
\end{aligned}
$$

where $\hat{\mathbf{t}}$ is a unit vector in isospin space, and $|0\rangle$ and $|I\rangle$ represent the ground and excited states of the system, with energies $\omega_{0}$ and $\omega_{I}$, respectively. SNM has zero isospin, and $R_{L}$ is therefore independent of the direction of $\hat{\mathbf{t}}$. The operator $O_{L}(\mathbf{q})$ represents the coupling of an external pion field to NM. In the case of PNM, we take $\hat{\mathbf{t}}$ to be in the z-direction, such that $\boldsymbol{\tau} \cdot \hat{\mathbf{t}}=-1$. $O_{L}(\mathbf{q})$ then represents the coupling of a $\pi^{0}$ field to PNM. Migdal 
assumed that this response would be dominated by a spin-isospin sound mode, and that the occurence of transition would be indicated by the vanishing of the corresponding excitation energy.

Calculation of the response of NM from realistic interactions is an extremely difficult task. However, it is well known [47] that the sums and energy-weighted sums of response functions are related to the two-body densities. For the IVSL response, the sum and energy-weighted sum are defined as:

$$
\begin{aligned}
A q^{2} S_{L}(q) & =\int_{0}^{\infty} R_{L}(q, \omega) d \omega \\
A q^{2} W_{L}(q) & =\int_{0}^{\infty} R_{L}(q, \omega) \omega d \omega
\end{aligned}
$$

thus removing the dependence on the number of particles. In SNM they are given by:

$$
\begin{aligned}
& S_{L}(q)=1+\frac{1}{9} \int\left[\rho_{2}^{\sigma \tau}(r) j_{0}(q r)-\rho_{2}^{t \tau}(r) j_{2}(q r)\right] d^{3} r \\
& W_{L}(q)=\frac{q^{2}}{2 m}+\frac{1}{2} \int\left[\sum_{p=2,6} D_{L}^{p}(r) \rho_{2}^{p}(r)\right] d^{3} r
\end{aligned}
$$

with the $D_{L}^{p}(r)$ tabulated in ref [47]. In the case of PNM we obtain:

$$
S_{L}(q)=1+\frac{1}{3} \int\left[\rho_{2}^{\sigma}(r) j_{0}(q r)-\rho_{2}^{t}(r) j_{2}(q r)\right] d^{3} r
$$

and

$$
\begin{aligned}
& D_{L}^{\sigma}(r)=-\frac{8}{3}\left[\tilde{v}^{\sigma}(r)\left(1-j_{0}(q r)\right)-\tilde{v}^{t}(r) j_{2}(q r)\right] \\
& D_{L}^{t}(r)=-\frac{4}{3}\left[\tilde{v}^{t}(r)\left(2+j_{0}(q r)\right)-\left(\tilde{v}^{\sigma}(r)-2 \tilde{v}^{t}(r)\right) j_{2}(q r)\right] .
\end{aligned}
$$

We note that terms with even $p$ do not occur in PNM, and the $\tilde{v}^{p}$ are defined as in Eq. (2.122.15). The energy-weighted sum of Eq. (5.6) contains only the contributions from the static parts of $v_{i j}$. These represent the dominant contributions to the energy-weighted sums of responses to electromagnetic fields [48,49, and presumably to $W_{L}$ as well.

The calculated values of $S_{L}(q)$ in SNM at $\rho=0.16 \mathrm{fm}^{-3}$ and $\rho=0.36 \mathrm{fm}^{-3}$, and in PNM at $\rho=0.16 \mathrm{fm}^{-3}$ and $\rho=0.2 \mathrm{fm}^{-3}$, appear in Fig. 11. At the higher densities, results 
obtained for both the LDP and HDP are shown. At equilibrium density, the $S_{L}(q)$ exhibits a small enhancement in the $q \sim 2 \mathrm{fm}^{-1}$ region. Indications of an enhancement of the IVSL response have been observed in $(\vec{p}, \vec{n})$ reactions [50]. In the LDP, this enhancement grows slowly with density. We predict a much larger enhancement at $q \sim 1.3 \mathrm{fm}^{-1}$ in the HDP.

When the response is dominated by a single collective mode, i.e. when only one of the states $|I\rangle$ largely contributes to the sum in Eq. (5.1), the energy of the collective state is given by $W_{L}(q) / S_{L}(q)$. As an example, the energy of Feynman phonons in atomic liquid ${ }^{4} \mathrm{He}$ can be obtained from the $W_{L} / S_{L}$ ratio. The spin-longitudinal response of nucleon matter probably has a large spread in energy; nevertheless, we can define a mean energy of the response as:

$$
\bar{E}_{L}(q)=\frac{W_{L}(q)}{S_{L}(q)} .
$$

The resulting values appear in Fig. 12 for the cases considered in Fig 11 .

We note that in the LDP, $\bar{E}_{L}(q)$ is larger than $q^{2} / 2 m$ in SNM as well as in PNM, indicating that the nuclear interactions push the response to higher energies on average. At $q \sim 0$ the IVSL response is almost entirely due to spin-isospin correlations, and $\bar{E}_{L}(q \sim 0)$ is therefore large. In Migdal's picture [18], the energy of the collective spin-isospin sound wave with $q \sim 1.3 \mathrm{fm}^{-3}$ decreases with increasing matter density, and pion condensation occurs when the energy vanishes. In the LDP, where $d_{t} \sim 4 r_{0}$, the present calculations show that $\bar{E}_{L}(q)$ does not decrease with density at any $q$. However, the HDP has a lower $\bar{E}_{L}(q)$ than the LDP in the vicinity of $q \sim 1.3 \mathrm{fm}^{-1}$. It is likely that a part of the response at $q \sim 1.3$ $\mathrm{fm}^{-1}$ shifts to lower energies, or softens, as the system moves from the LDP to the HDP.

\section{PIONIC INTERACTIONS AND EXCESS}

If the HDP is in fact a "pion-condensed" phase, it must have associated with it an enhanced pion field and enhanced pion exchange interactions between the nucleons. The interaction between two nucleons, due to the exchange of a pion of momentum $\mathbf{q}$, is given by: 


$$
v_{i j}^{\pi}(\mathbf{q})=-\frac{f_{\pi N N}^{2}}{m_{\pi}^{2}\left(m_{\pi}^{2}+q^{2}\right)} \boldsymbol{\sigma}_{\mathbf{i}} \cdot \mathbf{q} \boldsymbol{\sigma}_{\mathbf{j}} \cdot \mathbf{q} \boldsymbol{\tau}_{i} \cdot \boldsymbol{\tau}_{j} e^{i \mathbf{q} \cdot\left(\mathbf{r}_{\mathbf{i}}-\mathbf{r}_{\mathbf{j}}\right)} \Lambda^{2}(q),
$$

where $\Lambda(q)$ is the pion-nucleon form factor. The expectation value of the interaction is trivially related to the sum $S_{L}(q)$ of the IVSL response:

$$
\begin{aligned}
& \frac{1}{A}\left\langle\sum_{i<j} v_{i j}^{\pi}(\mathbf{q})\right\rangle=-\frac{f_{\pi N N}^{2}}{m_{\pi}^{2}\left(m_{\pi}^{2}+q^{2}\right)} \frac{3}{2} q^{2}\left(S_{L}(q)-1\right) \Lambda^{2}(q), \\
& \frac{1}{A}\left\langle\sum_{i<j} v_{i j}^{\pi}(\mathbf{q})\right\rangle=-\frac{f_{\pi N N}^{2}}{m_{\pi}^{2}\left(m_{\pi}^{2}+q^{2}\right)} \frac{1}{2} q^{2}\left(S_{L}(q)-1\right) \Lambda^{2}(q),
\end{aligned}
$$

in SNM and PNM, respectively. The expectation value of the complete $v_{i j}^{\pi}$ is obtained by integrating these expressions over dq:

$$
\begin{aligned}
\frac{1}{A}\left\langle\sum_{i<j} v_{i j}^{\pi}\right\rangle & =\frac{1}{2 A \pi^{2}} \int d q q^{2}\left\langle\sum_{i<j} v_{i j}^{\pi}(\mathbf{q})\right\rangle \\
& =\int d q \xi(q) .
\end{aligned}
$$

The function $\xi(q)$ :

$$
\xi(q)=\frac{1}{2 \pi^{2}} \frac{f_{\pi N N}^{2}}{m_{\pi}^{2}\left(m_{\pi}^{2}+q^{2}\right)} q^{4} \frac{3}{2}\left(1-S_{L}(q)\right) \Lambda^{2}(q)
$$

gives the pion-exchange nucleon-nucleon interaction (OPEP) contribution as a function of $\mathrm{q}$, the magnitude of the momentum of the exchanged pions in SNM; $\xi(q)$ for PNM is diminished by a factor of 3 . The calculated values of $\xi(q)$ for the cases previously discussed appear in Fig. 13 for SNM and PNM, where we have used:

$$
\Lambda\left(q^{2}\right)=\frac{\lambda^{2}}{\lambda^{2}+q^{2}}
$$

with $\lambda=5 \mathrm{fm}^{-1}$ as an illustration. In the LDP, the attraction from the OPEP comes from a broad region around $q \sim 3 \mathrm{fm}^{-1}$, whereas in the HDP, the attraction is relatively more concentrated at $q \sim 1.5 \mathrm{fm}^{-1}$. Also, much of the repulsion due to low $q$ pions in the LDP is absent in the HDP. The total OPEP contribution, obtained from Eq. (6.4), is listed in Table [II]. In the case of SNM, the $\left\langle v^{\pi}\right\rangle$ is not very different in the LDP and HDP; a much larger change occurs in the $\left\langle V^{2 \pi}\right\rangle$, also listed in table $\mathbb{1 1}$. In PNM, however, the difference in $\left\langle v^{\pi}\right\rangle$ is more pronounced between the LDP and the HDP. 
The difference in the expectation values of the pion number operator in matter and for $\mathrm{A}$ isolated nucleons is called the pion excess [51]. The operator for excess pions of momentum q, due exclusively to OPE interactions, is given by:

$$
\delta n_{\pi}^{(1)}(q)=-\frac{v^{\pi}(q)}{\sqrt{ } m_{\pi}^{2}+q^{2}} .
$$

The distribution of excess pions is given by:

$$
\eta(q)=-\frac{\xi(q)}{\sqrt{ } m_{\pi}^{2}+q^{2}},
$$

which is shown in Fig. 14 for SNM and PNM. The $\eta(q)$ exhibits a sharp enhancement in the HDP at $q \sim 1.5 \mathrm{fm}^{-1}$.

The integral of $\delta n_{\pi}^{(1)}(q)$, denoted by $\left\langle\delta n_{\pi}^{(1)}\right\rangle$, is listed in Table [11. The total $\left\langle\delta n_{\pi}\right\rangle$ includes other contributions from the $\mathrm{NN} \rightarrow \mathrm{N} \Delta$ and $\mathrm{NN} \rightarrow \Delta \Delta$ transition interactions [51], which we have not calculated here. These additional contributions take into account the changes in the pion field due to $\mathrm{N} \Delta$ - and $\Delta \Delta$-box diagrams and due to the $V_{i j k}^{2 \pi}$.

\section{CONCLUSIONS}

We have studied the short-range spin-isospin structure of SNM and PNM, using one of the most accurate models of nuclear forces currently available. For SNM at equilibrium density, we predict a short-range structure very similar to that found by Forest et al. [7] in light nuclei, at interparticle distances $<1.5 \mathrm{fm}$. Symmetric Nuclear Matter is bound by small localized regions of strong attraction in the $\mathrm{NN}$ potential in $\mathrm{T}, \mathrm{S}=0,1$ and 1,0 states. The two-nucleon densities are found to have large overshoots, ranging up to 2.5 (1.5) times the uncorrelated values (Figs. 6 and 7 ) in the T,S $=0,1(1,0)$ attractive regions. The interaction, and consequently the pair density, in $\mathrm{T}, \mathrm{S}=0,1$ states is highly anisotropic due to the presence of the OPE tensor force. The two-body density overshoots in this state have femtometer-sized toroidal structures similar to those found in light nuclei; we therefore expect this feature to occur in all nuclei. These short-range structures are not very sensitive to the uncertainties in models of nuclear forces as discussed in Reference [7]. 
We also find that the Argonne $v_{18}$ plus Urbana IX $V_{i j k}$ model of nuclear forces, which offers one of the best fits to the Nijmegen NN scattering database, as well as to the binding energies of light nuclei [26], predicts that both SNM and PNM will undergo transitions to phases with pion condensation at densities of $\sim 0.32 \mathrm{fm}^{-3}$ and $\sim 0.2 \mathrm{fm}^{-3}$, respectively. The occurrence of this transition is sensitive to the short-range parts of the $\boldsymbol{\sigma}_{i} \cdot \boldsymbol{\sigma}_{j}$ and $\boldsymbol{\sigma}_{i} \cdot \boldsymbol{\sigma}_{j} \boldsymbol{\tau}_{i} \cdot \boldsymbol{\tau}_{j}$ NN interaction, as predicted by Migdal [18. The transition does not occur with the older Urbana and Argonne $v_{14}$ models in SNM, while in PNM it occurs with the Argonne $v_{14}$, though not with the Urbana $v_{14}$.

It should be stressed that the present calculations of pion condensation in the HDP are incomplete. Although we have used a variational wavefunction of the same form to describe both the LDP and the HDP, a different form permitting long-range order should be used for the latter. A wavefunction describing a correlated liquid crystal, containing layers of spin-isospin ordered nucleons, may be more appropriate for the HDP. Takatsuka et al. [52 have used such a wavefunction, denoted ASL for "alternating spin layers". A correlated spin-ordered solid (SOS) wavefunction [53] has also been used in the past. However, only lowest-order variational [53] or G-matrix [52] calculations have been possible with these wavefunctions having long-range order. In view of the small difference between the energies of the LDP, and the HDP, it may be advisable to use similar methods to calculate both. The chain summation methods used here cannot be used with either ASL or SOS wavefunctions. However, in future it may be possible to calculate energies of the LDP as well as correlated ASL and SOS phases, using cluster Monte Carlo methods [6, 35, 8].

The indication of a phase transition obtained with the present calculation should be reliable. The changes in the pair distributions, the IVSL response and the pion fields strongly suggest that the HDP will exhibit pion condensation upon inclusion of wavefunctions permitting long-range order. We hope that such a modification will not significantly alter our results for the short-range structure and energy of the HDP. The momentum of the condensed pion field is $\sim 1.4 \mathrm{fm}^{-1}$ at the onset of the phase transition, which corresponds to a wavelenth of $4.5 \mathrm{fm}$. The interlayer spacing, which equals half the wavelength [54], is thus 
predicted to be $\sim 2.25 \mathrm{fm}$ in both $\mathrm{SNM}$ and PNM. This spacing is larger than the spacing of 1.5 (1.7) fm between the layers of a simple cubic solid at $\rho=0.32(0.2) \mathrm{fm}^{-3}$.

Most calculations exploring the possibility of pion condensation in matter explicitly consider the $\pi \mathrm{N} \Delta$ coupling. The baryons in ASL and SOS matter are taken to be superpositions of nucleon and $\Delta$-states. The $\mathrm{NN} \rightarrow \mathrm{N} \Delta$ and $\mathrm{NN} \rightarrow \Delta \Delta$ transitions are considered in these approaches, which make the theory more difficult. In contrast, we do not consider the $\Delta$ degree of freedom explicitly in the present work. The resulting effects, along with those due to other mesons and nucleon resonances, are implicit in the two- and three-nucleon potentials obtained by fitting experimental data. Our Hamiltonian has only nucleon degrees of freedom, and its predictions can be calculated using a variety of many-body techniques. As previously mentioned, however, the predictions regarding pion condensations are sensitive to the details of the short-range NN interaction.

It is encouraging to note that our calculated density of $\sim 0.32 \mathrm{fm}^{-3}$ for the onset of pion condensation in SNM is within the range 0.32 to $0.48 \mathrm{fm}^{-3}$ favored by calculations using the ASL wavefunction [52]. As with WFF, we predict a lower transition density $\left(\sim 0.2 \mathrm{fm}^{-3}\right)$ for PNM. This is due to the fact that in most realistic models, $\tilde{v}^{\sigma}(r)$, the relevant interaction in PNM, is softer than $v^{\sigma \tau}(r)$ (Fig. 10). Calculations with ASL wavefunctions predict a higher density of $\sim 0.5 \mathrm{fm}^{-3}$ for the onset of neutral pion condensation in PNM [52]. The possibility of charged $\pi^{-}$and/or $K^{-}$condensation in neutron star matter at densities above

our result of $0.2 \mathrm{fm}^{-3}$ for $\pi^{0}$ condensation is currently being investigated by a number of researchers 55 57.

\section{ACKNOWLEDGMENTS}

The authors thank J.L. Forest, S.C. Pieper and R.B. Wiringa for many discussions and useful suggestions, D.S. Lewart for help preparing the manuscript and figures, and C.M. Elliot for proofreading the manuscript. This work was supported by the U.S. National Science Foundation via grant PHY 94-21309. Calculations were performed on the Cray C90 
at the Pittsburgh Supercomputing Center.

\section{APPENDIX A: THE TREATMENT OF $L^{2}$ AND $(\mathbf{L} \cdot \mathbf{S})^{2}$ INTERACTIONS}

The leading contribution to $\langle v\rangle-\mathrm{MB}-\mathrm{q}$ is from the separable, direct three-body diagram shown in Fig. 15a. The earlier calculation of $\langle v\rangle$-MB-q by Lagaris [11] with the U14 potential included only the main term of this diagram, namely the term with only central correlations between interacting particles. While this approximation was justifiable for U14, the much stronger $L^{2}$ and $(\mathbf{L} \cdot \mathbf{S})^{2}$ interactions of A18 (see Table I) require calculation of additional terms. In the present work we include all relevant terms of the direct diagram presented in Fig. 15]a, and in addition consider: i) the interacting exchange three-body separable diagram (Fig. 15b), ii) the passive exchange three-body separable diagram (Fig. 15c), and iii)central chain diagrams with and without exchanges (Figs. 15d-g.) Terms in these diagrams are classified as either F-diagrams, in which the gradients in the interaction operate on passive correlations $F_{i l}$, and K-diagrams, in which the gradients act on interacting correlations $F_{i j}$ and the uncorrelated wavefunction $\Phi$. The direct three-body separable diagram (15a), has the general form:

$$
\begin{aligned}
& \frac{1}{A \Omega^{2}} \sum_{\mathbf{k}_{i}, \mathbf{k}_{j}, \mathbf{k}_{l}} \sum_{p, m, p^{\prime}, q, q^{\prime}} \int \Phi_{3}^{*}(i, j, l) C\left[\frac{1}{4}\left\{f_{i j}^{p} O_{i j}^{p}, f_{i l}^{q} O_{i l}^{q}\right\} v_{i j}^{m} O_{i j}^{m}\left\{f_{i j}^{p^{\prime}} O_{i j}^{p^{\prime}}, f_{i l}^{q^{\prime}} O_{i l}^{q^{\prime}}\right\}\right. \\
&\left.-\left(f_{i j}^{p} O_{i j}^{p} v_{i j}^{m} O_{i j}^{m} f_{i j}^{p^{\prime}} O_{i j}^{p^{\prime}}\right)\left(f_{i l}^{q} O_{i l}^{q} f_{i l}^{q^{\prime}} O_{i l}^{q^{\prime}}\right)\right] \Phi_{3}(i, j, l) d^{3} r_{i j} d^{3} r_{i l},
\end{aligned}
$$

with

$$
\Phi_{3}(i, j, l)=e^{i\left(\mathbf{k}_{i} \cdot \mathbf{r}_{i}+\mathbf{k}_{j} \cdot \mathbf{r}_{j}+\mathbf{k}_{l} \cdot \mathbf{r}_{l}\right)}
$$

where C[ ] represents the so-called c-part, or the $\boldsymbol{\sigma}, \boldsymbol{\tau}$-independent part of the operator product, as described in Reference [3]. Also, the $O_{i j}$ in the separated part of the expression do not operate on the $f_{i l} O_{i l}$. The expressions for the interacting and passive exchange diagrams (Figs. 15a and b) are obtained by replacing the uncorrelated wavefunction $\Phi_{3}^{*}(i, j, l)$ with

either $\Phi_{3}^{*}(j, i, l)$ or $\Phi_{3}^{*}(l, j, i)$, and inserting the appropriate exchange operators $\left(\frac{1}{4} \sum_{n=1,4} O_{i j}^{n}\right.$ or $\left.\frac{1}{4} \sum_{n=1,4} O_{i l}^{n}\right)$ to the far left of the operator product. 
The three-body separable $L^{2}$ diagrams with $m=9-12$ (Figs. 15a-c) have large F-parts, and smaller K-parts which we neglect. For the diagram presented in Fig. 15a we include terms with $p, p^{\prime}=1-6$ and $q, q^{\prime}=1-6$. The dominant contribution comes from terms with $p=1$ and $q, q^{\prime}$ representing either central or tensor correlations. The contribution from the diagrams presented in Figs. 15b and 15kc is somewhat smaller, and we only consider terms with $p, p^{\prime}=1,2$ and $q, q^{\prime}=1-6$. Contributions to the energy of SNM at $\rho=0.28 \mathrm{fm}^{-3}$ for both the U14 and A18 models, without three-body interactions are listed in Table एV.

The $(\mathbf{L} \cdot \mathbf{S})_{i j}^{2}$ in $O_{i j}^{m=13,14}$ can be decomposed as:

$$
(\mathbf{L} \cdot \mathbf{S})_{i j}^{2}=-\frac{1}{2}(\mathbf{L} \cdot \mathbf{S})_{i j}+\frac{1}{2} L^{2}+\frac{1}{6} \boldsymbol{\sigma}_{i} \cdot \boldsymbol{\sigma}_{j} L^{2}+\frac{1}{6} \alpha_{i j}(L, L),
$$

where:

$$
\alpha_{i j}(L, L)=3 \boldsymbol{\sigma}_{i} \cdot \mathbf{L} \boldsymbol{\sigma}_{j} \cdot \mathbf{L}-\boldsymbol{\sigma}_{i} \cdot \boldsymbol{\sigma}_{j} L^{2} .
$$

The $(\mathbf{L} \cdot \mathbf{S})_{i j}$ part of $v_{i j}^{m=13,14}$ is treated along with the $v_{i j}^{m=7,8}$, as described by Lagaris [31, and includes all the diagrammatic terms therein. The $L^{2}$ parts are calculated along with the $v_{i j}^{m=9-12}$, as described above. We also include selected $\alpha_{i j}$ diagrams, which are expected to make the most significant contribution to the energy. These contain direct F-diagrams (Fig. 15a) with: $p^{\prime} q^{\prime} p q=c c t c, t c c c, t t c t, c t t t, t c t c$, and the exchange F-diagrams (Fig. 15b) with $p^{\prime} q^{\prime} p q=c c t c, t c c c$, where it is understood that ' $t$ ' represents all tensor correlations with and without $\boldsymbol{\tau}_{i} \cdot \boldsymbol{\tau}_{j}$ or $\boldsymbol{\tau}_{i} \cdot \boldsymbol{\tau}_{l}$ factors.

The central chain diagrams shown in Figs. 15d d-g make a modest contribution to the energy. The K-diagrams are obtained by inserting appropriate chain functions into the twobody integrals. Three central chain functions, $G_{x x^{\prime}=d d, d e, c c}^{c}$ are defined in Reference [3], where $x x^{\prime}$ denote the exchange character of the two interacting vertices of the diagram. Each of these may be either direct $(x=d)$, exchanged only with particles in the chain $(x=e)$ or part of a circular exchange involving both interacting particles $\left(x x^{\prime}=c c\right)$. When considering only static interactions and correlations, the $G_{x x^{\prime}}^{c}$ can be directly inserted into the twobody integrals. However, the gradients associated with momentum-dependent interactions 
may operate on the Slater functions in the de and cc chains, thus yielding somewhat more complicated expressions.

The direct central chain diagram (Fig. 15/d) has neither interacting particle exchanged. The K-part of this diagram is obtained in the same manner as static diagrams, namely by dressing the two-body direct diagram with $\left[e^{G_{d d}^{c}}-1\right]$.

In order to calculate the de and cc diagrams (Figs. $15 \mathrm{f}$ and $15 \mathrm{~g}$ ), the operator product $f_{i j}^{p} O_{i j}^{p} v_{i j}^{m} O_{i j}^{m} f_{i j}^{p^{\prime}} O_{i j}^{p^{\prime}}$ is written in powers of $L^{2}$. The $L^{0}$ part does not contain gradients, and the associated de and ee chain diagrams are thus obtained as in the static case by dressing the direct two-body diagram with:

$$
e^{G_{d d}^{c}}\left(2 G_{d e}^{c}+G_{d e}^{c} G_{d e}^{c}+G_{e e}^{c}\right)
$$

The $L^{0}$ cc chain diagrams (Figs. 15e and 15g) are calculated by replacing $l_{i j}^{2} / s$ in the two-body exchange diagram by:

$$
\frac{1}{s} e^{G_{d d}^{c}}\left(l_{i j}+s G_{c c}^{c}\right)^{2}-\frac{l_{i j}^{2}}{s} .
$$

Here, $s$ is the degeneracy, and $l_{i j} \equiv l\left(k_{f} r_{i j}\right)$ is the Slater function. The K-contribution of terms in the de and cc diagrams containing $L^{2}$ operators are calculated using the chain functions $G_{d e}^{L^{2}}$ and $G_{c c}^{L^{2}}$ defined as:

$$
\begin{aligned}
G_{d e}^{L^{2}} & =-\frac{\rho r_{i j}^{2}}{s} \int d^{3} r_{l} F_{l j}^{c} l_{i l} \tilde{l}_{i l} \\
G_{c c}^{L^{2}} & =-\frac{\rho r_{i j}^{2}}{s^{2}} l_{i j} \int d^{3} r_{l} F_{l j}^{c} l_{l j} \tilde{l}_{i l}
\end{aligned}
$$

with

$$
\tilde{l}_{i l} \equiv \frac{1}{4} \cos ^{2} \theta_{i}\left(l_{i l}^{\prime \prime}-\frac{l_{i l}^{\prime}}{r_{i l}}\right)-\frac{1}{4}\left(l_{i l}^{\prime \prime}+\frac{l_{i l}^{\prime}}{r_{i l}}\right)+\cos \theta_{i} \frac{l_{i l}^{\prime}}{r_{i j}} .
$$

The $L^{2}$ chain functions result from the $L_{i j}^{2}$ acting on the Fermi gas part of the wavefunctions. These depend on $r_{i}$ and $r_{j}$ due to the exchanges at the interacting vertices of the chain diagrams.

The de contribution of the $L^{2}$ part is obtained by dressing the two-body direct diagram with: 


$$
e^{G_{d d}^{c}}\left(G_{d e}^{c}+2\left(5 / k_{f}^{2} r_{i j}^{2}\right) G_{d e}^{L^{2}}\right)
$$

while the cc contribution is calculated by replacing $r_{i j} l_{i j} l_{i j}^{\prime} / s$ in the two-body exchange diagram by:

$$
e^{G_{d d}^{c}}\left(\frac{r_{i j} l_{i j} l_{i j}^{\prime}}{s}-r_{i j} l_{i j}^{\prime} G_{c c}^{c}+2 G_{c c}^{L^{2}}\right)-\frac{r_{i j} l_{i j} l_{i j}^{\prime}}{s}
$$

The present calculation of $\langle v\rangle$-MB-q includes terms involving only the static correlations, since they yield the dominant contribution to the energy. Without $(\mathbf{L} \cdot \mathbf{S})_{i j}$ correlations, the $f_{i j}^{p} O_{i j}^{p} v_{i j}^{m} O_{i j}^{m} f_{i j}^{p^{\prime}} O_{i j}^{p^{\prime}}$ operator product does not contain terms with $L^{n>2}$, and the above calculation of central chain K-diagrams is complete.

\section{APPENDIX B: PERTURBATIVE CORRECTIONS}

We wish to calculate the two-body cluster using a better variational wavefunction. The standard two-body variational wavefunction has the form:

$$
\Psi_{2 b}=\left(\sum_{p=1,8} f^{p}\left(r_{i j}\right) O_{i j}^{p}\right) \Phi_{2 b},
$$

where $\Phi_{2 b}$ is a plane-wave Slater determinant. The $f^{p}$ are determined from the E-L equations obtained by minimizing the total two-body cluster energy

$$
C_{2}=\frac{1}{A} \sum_{m, n}\left\langle\Psi_{2 b}\left|H-\frac{\hbar^{2}}{m} k_{m n}^{2}\right| \Psi_{2 b}\right\rangle=\frac{1}{A} \sum_{\mathbf{k}_{m}, \mathbf{k}_{n}} \sum_{\boldsymbol{\sigma}_{m}, \boldsymbol{\sigma}_{n}} \sum_{\boldsymbol{\tau}_{m}, \boldsymbol{\tau}_{n}}\left\langle\Psi_{2 b}\left|H-\frac{\hbar^{2}}{m} k_{m n}^{2}\right| \Psi_{2 b}\right\rangle,
$$

obtained after summing over $\mathbf{k}_{m}, \mathbf{k}_{n}, \boldsymbol{\sigma}_{m}, \boldsymbol{\sigma}_{n}, \boldsymbol{\tau}_{m}$ and $\boldsymbol{\tau}_{n}$ at chosen values of $d_{c}, d_{t}$ and $\alpha$. We propose instead to minimize $C_{2}\left(k_{m}, k_{n}\right)$ separately in each partial wave, thus obtaining momentum- and channel-dependent correlations $f(l, S, J, k)$. The perturbative correction to the energy in an $l, S, J$ channel is then given by:

$$
\delta E_{2}(l, S, J)=\sum_{\mathbf{k}_{m}, \mathbf{k}_{n}}\left[C_{2}\left(l, S, J ; k_{m}, k_{n}\right)-\bar{C}_{2}\left(l, S, J ; k_{m}, k_{n}\right)\right],
$$

where $C_{2}$ is obtained from the $f(l, S, J, k)$ and $\bar{C}_{2}$ is calculated with the operator $f^{p} O^{p}$.

The uncorrelated two-body wavefunction, in relative coordinates, can be expanded as: 


$$
\begin{aligned}
e^{i k z}|S, M\rangle & =\delta_{S 0} \delta_{M 0} \sum_{J} \sqrt{4 \pi(2 J+1)} i^{J} j_{J}(k r) \mathcal{Y}_{J 0 J}^{0} \\
& +\delta_{S 1} \sum_{J} \sqrt{4 \pi(2 J+1)} i^{J} j_{J}(k r) \mathcal{Y}_{J 1 J}^{M}\langle J 1 J M \mid J 01 M\rangle \\
& +\delta_{S 1} \sum_{J \geq 1} \sqrt{4 \pi} i^{J_{-}}\left[\xi_{J_{-}}^{M}(r) \mathcal{Y}_{J_{-} 1 J}^{M}+\xi_{J_{+}}^{M}(r) \mathcal{Y}_{J_{+} 1 J}^{M}\right],
\end{aligned}
$$

where $J_{ \pm}=J \pm 1, \mathcal{Y}_{l S J}^{M}$ are spin-angle functions, $\left\langle l S J M \mid l m_{l} S m_{s}\right\rangle$ are Clebsch-Gordon coefficients and:

$$
\begin{aligned}
& \xi_{J_{-}}^{M}(r)=\sqrt{\left(2 J_{-}+1\right)}\left\langle J_{-} 1 J M \mid J_{-} 01 M\right\rangle j_{J_{-}}(k r) \\
& \xi_{J_{+}}^{M}(r)=-\sqrt{\left(2 J_{+}+1\right)}\left\langle J_{+} 1 J M \mid J_{+} 01 M\right\rangle j_{J_{+}}(k r) .
\end{aligned}
$$

The correlated two-body wavefunction can thus be written:

$$
\begin{aligned}
\Psi(r, k, S, M) & =\sum_{J} \sqrt{4 \pi(2 J+1)} i^{J}\left[R_{J, S=0}(r) \mathcal{Y}_{J 0 J}^{0}+R_{J, S=1}(r) \mathcal{Y}_{J 1 J}^{M}\langle J 1 J M \mid J 01 M\rangle\right] \\
& +\sum_{J \geq 1} \sqrt{4 \pi} i^{J_{-}}\left[R_{J_{-}}^{M}(r) \mathcal{Y}_{J_{-} 1 J}^{M}+R_{J_{+}}^{M}(r) \mathcal{Y}_{J_{+} 1 J}^{M}\right] .
\end{aligned}
$$

The wavefunction $u_{J=l, S=0,1}=r R_{J, S}$ in uncoupled channels satisfies the E-L equation:

$$
-\frac{\hbar^{2}}{m} u_{J, S}^{\prime \prime}+\left[\frac{\hbar^{2}}{m} \frac{J(J+1)}{r^{2}}+\bar{v}_{J, S}-\lambda_{J, S}(k)\right] u_{J, S}=\frac{\hbar^{2}}{m} k^{2} u_{J, S}
$$

and is normalized such that:

$$
u_{J, S}\left(r=d_{x}\right)=r j_{J}(k r)
$$

The $\lambda_{J, S}(k)$ are constants, which are varied to match the boundary conditions:

$$
u_{J, S}^{\prime}\left(r=d_{x}\right)=j_{J}(k r)+r j_{J}^{\prime}(k r),
$$

where $d_{x}=d_{c}$ in the spin-singlet state, and $d_{x}=d_{t}$ in the uncoupled spin-triplet state, which is affected by the tensor correlations.

In the coupled channels, $J=l \pm 1, S=1$, we obtain a pair of coupled equations:

$$
\begin{aligned}
& -\frac{\hbar^{2}}{m} u^{\prime \prime}+\left[\frac{\hbar^{2}}{m} \frac{J_{-}\left(J_{-}+1\right)}{r^{2}}+\bar{v}_{J_{-}, 1}-\lambda^{c}(k)\right] u+\frac{6 \sqrt{J(J+1)}}{2 J+1}\left(\bar{v}_{J, 1}^{t}-\lambda^{t}(k)\right) \omega=\frac{\hbar^{2}}{m} k^{2} u \\
& -\frac{\hbar^{2}}{m} \omega^{\prime \prime}+\left[\frac{\hbar^{2}}{m} \frac{J_{+}\left(J_{+}+1\right)}{r^{2}}+\bar{v}_{J_{+}, 1}-\lambda^{c}(k)\right] \omega+\frac{6 \sqrt{J(J+1)}}{2 J+1}\left(\bar{v}_{J, 1}^{t}-\lambda^{t}(k)\right) u=\frac{\hbar^{2}}{m} k^{2} \omega .
\end{aligned}
$$


These equations have two sets of solutions, denoted by $\left(u_{-}, \omega_{-}\right)$and $\left(u_{+}, \omega_{+}\right)$, with boundary conditions:

$$
\begin{aligned}
& u_{-}\left(r=d_{x}\right)=r j_{J_{-}}(k r) ; \omega_{-}\left(r=d_{t}\right)=0, \\
& u_{-}^{\prime}\left(r=d_{x}\right)=j_{J_{-}}(k r)+r j_{J_{-}}^{\prime}(k r) ; \omega_{-}^{\prime}\left(r=d_{t}\right)=0,
\end{aligned}
$$

where $d_{x}=d_{c}$ for $J=1, l=J_{-}=0,{ }^{3} S_{1}$ state, and $d_{t}$ otherwise, and

$$
\begin{aligned}
& u_{+}\left(r=d_{t}\right)=0 ; \omega_{+}\left(r=d_{t}\right)=r j_{J_{+}}(k r), \\
& u_{+}^{\prime}\left(r=d_{t}\right)=0 ; \omega_{+}^{\prime}\left(r=d_{t}\right)=j_{J_{+}}(k r)+r j_{J_{+}}^{\prime}(k r) .
\end{aligned}
$$

The $\lambda^{c}(k)$, is adjusted to match the derivative boundary condition on the dominant wave $\left(u_{-}\right.$or $\left.\omega_{+}\right)$, while $\lambda^{t}(k)$ is varied to match the zero derivative condition on the secondary wave $\left(u_{+}\right.$and $\left.\omega_{-}\right)$. Thus, $\lambda^{c}(k)$ and $\lambda^{t}(k)$ depend on $J$ and the $l$ of the dominant wave.

The $R_{J_{ \pm}}^{M}$ can be expressed as superpositions of the two solutions, which match the boundary condition: $R_{J_{ \pm}}^{M}(r \rightarrow \infty)=\xi_{J_{ \pm}}^{M}(r \rightarrow \infty)$. Evaluating the Clebsch-Gordon coefficients in $\xi_{J_{ \pm}}^{M}$, we find:

$$
\begin{aligned}
R_{J_{-}}^{M= \pm 1} & =\frac{1}{r}\left[u_{-} \sqrt{\frac{J+1}{2}}-u_{+} \sqrt{\frac{J}{2}}\right] \\
R_{J_{+}}^{M= \pm 1} & =\frac{1}{r}\left[\omega_{-} \sqrt{\frac{J+1}{2}}-\omega_{+} \sqrt{\frac{J}{2}}\right] \\
R_{J_{-}}^{M=0} & =\frac{1}{r}\left[u_{-} \sqrt{J}+u_{+} \sqrt{J+1}\right] \\
R_{J_{-}}^{M=0} & =\frac{1}{r}\left[\omega_{-} \sqrt{J}+\omega_{+} \sqrt{J+1}\right] .
\end{aligned}
$$

With the wavefunction in hand, we can calculate the two-body cluster energy, and thereby determine $\delta E_{2}(l, S, J)$. The differential equations for the wavefunctions are solved at several values of $k$ on a grid from 0 to $k_{f}$. The expressions for $C_{2}\left(l, S, J, k_{m}, k_{n}\right)$ are then integrated over the Fermi sea. In order to insure that the correlations functions, $f$, in coupled channels are positive, the solutions are matched to the asymptotic forms at the first node of the bessel function, rather than at the healing distance $\left(d_{c}\right.$ or $\left.d_{t}\right)$ for values of $k$ large enough such that the node occurs within the healing distance. 
The momentum-dependence of $f(l, S, J, k)$ is not very large, however the dependence on $l, S, J$ channels is significant. We find that no additional attraction is obtained in the ${ }^{3} S_{1}-{ }^{3} D_{1}$ channel, and very little comes from the ${ }^{1} S_{0}$ and other singlet channels as can be seen in Table $\nabla$. This presumably indicates that the parameters $d_{c}, d_{t}$ and $\alpha$ are optimum for those channels. The bulk of the additional attraction due to channel- and momentumdependent correlations is in the ${ }^{3} P_{2}-{ }^{3} F_{2}$ and ${ }^{3} P_{1}$ channels, with somewhat less coming from the ${ }^{3} D_{2}$ channel. The present $f^{p} O^{p}$ correlation operator, with $d^{p}$ chosen according to Eqs. 3.8 and 3.9, is probably inadequate to simultaneously describe correlations in $S$ - and $P$-waves accurately. However, the $\delta E_{2}$ correction has little effect on the critical density of the predicted phase transition. 


\section{REFERENCES}

* Electronic address: aakmal@uiuc.edu

$\dagger \quad$ Electronic address: vijay@rsm1.physics.uiuc.edu

[1] K.A. Brueckner, C.A. Levinson, and H.M. Mahmoud, Phys. Rev. 95, 217 (1954).

[2] H. A. Bethe, Annu. Rev. Nucl. Sci. 21, 93 (1971).

[3] V. R. Pandharipande and R. B. Wiringa, Rev. Mod. Phys. 51, 821 (1979).

[4] B. D. Day, Phys. Rev. Lett. 47, 226 (1981).

[5] B. D. Day and R. B. Wiringa, Phys. Rev. C 32, 1057 (1985).

[6] S. C. Pieper, R. B. Wiringa and V. R. Pandharipande, Phys. Rev. C 46, 1741 (1992).

[7] J. L. Forest, V.R. Pandharipande, S.C. Pieper, R.B. Wiringa, R. Schiavilla and A. Arriaga, Phys. Rev. C 54, 646 (1996).

[8] B. S. Pudliner, A. Smerzi, J. Carlson, V. R. Pandharipande, S. C. Pieper and D. G. Ravenhall, Phys. Rev. Lett. 76, 2416 (1996).

[9] B. Friedman and V. R. Pandharipande, Nucl. Phys. A361, 501 (1981).

[10] I. E. Lagaris and V. R. Pandharipande, Nucl. Phys. A359 331, (1981).

[11] I. E. Lagaris and V. R. Pandharipande, Nucl. Phys. A359 349, (1981).

[12] I. E. Lagaris and V. R. Pandharipande, Nucl. Phys. A369, 470 (1981).

[13] J. Lomnitz-Adler, V. R. Pandharipande and R. A. Smith, Nucl. Phys. A361, 399 (1981).

[14] J. Fujita and H. Miyazawa, Prog. Theor. Phys. 17, 360 (1957).

[15] R. Schiavilla, V. R. Pandharipande and R. B. Wiringa, Nucl. Phys A449, 219 (1986).

[16] R. B. Wiringa, V. Fiks and A. Fabrocini, Phys. Rev. C38, 1010 (1988). 
[17] R. B. Wiringa, R. A. Smith and T. L. Ainsworth, Phys. Rev. C 29, 1207 (1984).

[18] A. B. Migdal, Rev. Mod. Phys. 50, 107 (1978).

[19] R.F. Sawyer and D.J. Scallapino, Phys. Rev. D7, 953 (1973).

[20] T. Kunihiro, T. Muto, T. Takatsuka, R. Tamagaki and T. Tatsumi, Prog. Theor. Phys. supplement 112, 1 (1993)

[21] C. J. Pethick, Rev. Mod. Phys. 64, 1133 (1992)

[22] J. Carlson, Phys. Rev. C 38, 1879 (1988).

[23] R. B. Wiringa, V. G. J. Stoks and R. Schiavilla, Phys. Rev. C 51, 38 (1995).

[24] J.R. Bergervoet, P.C. van Campen, R.A.M. Klomp, J.-L. de Kok, T.A. Rijken, V.G.J. Stoks, and J.J. de Swaart, Phys. Rev. C 41, 1435 (1990).

[25] V.G.J. Stoks, R.A.M. Klomp, M.C.M. Rentsmeester, and J.J. de Swaart, Phys. Rev. C 48, $792(1993)$.

[26] B.S. Pudliner, V.R. Pandharipande, J. Carlson, and R.B. Wiringa, Phys. Rev. Lett. 74, 4396 (1995).

[27] B.S. Pudliner, Ph.D. Thesis, University of Illinois at Urbana-Champaign (1996).

[28] B.S. Pudliner, V.R. Pandharipande, J. Carlson, S.C. Pieper and R.B. Wiringa, Phys. Rev. C 56, 1720 (1997).

[29] R.B. Wiringa, Nucl. Phys. A401, 86 (1983).

[30] S.A. Coon, M.D. Scadron, P.C. McNamee, B.R. Barrett, D.W.E. Blatt and B.H.J. McKellar, Nucl. Phys. A317, 242 (1979).

[31] I. E. Lagaris and V. R. Pandharipande, Nucl. Phys. A334, 217 (1980).

[32] A. Arriaga, V.R. Pandharipande and R.B. Wiringa, Phys. Rev. C 52, 2362 (1995). 
[33] R.B. Wiringa, private communication (1997).

[34] R.B. Wiringa and V.R. Pandharipande, Phys. Lett. 99B, 1 (1981).

[35] S.C. Pieper, private communication (1997).

[36] P.J. Siemens and V.R. Pandharipande, Nucl. Phys. A173, 561 (1971).

[37] V.R. Pandharipande, Nucl. Phys. A178, 123 (1971).

[38] Q.N. Usmani, B. Friedman and V.R. Pandharipande, Phys. Rev. B25, 4502 (1982).

[39] J. Carlson, V. R. Pandharipande and R. B. Wiringa, Nucl. Phys. A401 59 (1983).

[40] V.R. Pandharipande and H.A. Bethe, Phys. Rev. C 7, 1312 (1973).

[41] S. Fantoni, B. L. Friman and V. R. Pandharipande, Nucl. Phys. A399, 57 (1983).

[42] A. Fabrocini and S. Fantoni, Phys. Lett. B298, 263 (1993).

[43] R. Machleidt, K. Holinde, and Ch. Elster, Phys. Rep. 149, 1 (1987).

[44] R. Machleidt, Adv. Nucl. Phys. 19, 189 (1989).

[45] M. Lacombe, B. Loiseau, J.M. Richard, R. Vinh Mau, J. Côté, P. Pirès, and R. de Tourreil, Phys. Rev. C 21, 861 (1980).

[46] V.R. Pandharipande, Nucl. Phys. A553, 191c (1993)

[47] V. R. Pandharipande, J. Carlson, S.C. Pieper, R.B. Wiringa and R. Schiavilla, Phys. Rev. C49, 789 (1994).

[48] R. Schiavilla, A. Fabrocini and V.R. Pandharipande, Nucl. Phys. A473, 290 (1987).

[49] R. Schiavilla, Nucl. Phys. A499, 301 (1989).

[50] T.N. Taddeucci et al., Phys. Rev. Lett 73, 3516 (1994).

[51] B. L. Friman, V. R. Pandharipande and R. B. Wiringa, Phys. Rev. Lett. 51, 763 (1983). 
[52] T. Takatsuka, K. Tamiya, T. Tatsumi, and R. Tamagaki, Prog. Theor. Phys. 59, 1933 (1978).

[53] V.R. Pandharipande and R.A. Smith, Nucl. Phys. A237, 507 (1975).

[54] V.R. Pandharipande, in Astrophysics and Gravitation, Proc. of 16th Solvay Conf. in Phys. on Astrophysics and Gravitation, Univ. of Brussels (1974).

[55] T. Takatsuka, R. Tamagaki and T. Tatsumi, Prog. Theor. Phys. Supplement 112, 67 (1993)

[56] G. Brown, C. Lee, M. Rho, V. Thorsson, Nucl. Phys. A 572, 693 (1994).

[57] V.R. Pandharipande, C.J. Pethick and V. Thorsson, Phys. Rev. Lett. 75, 4567 (1995). 


\section{FIGURES}

FIG. 1. $E(\rho)$ of SNM calculated using A18+UIX. Included for comparison are previous calculations of $E(\rho)$ using A14+UVII (WFF), and U-DDI (FP). The two sets of variational minima obtained at $\rho>0.28 \mathrm{fm}^{-3}$ are labeled LDP and HDP.

FIG. 2. $E(\rho)$ of PNM calculated using A18+UIX. Included for comparison are previous calculations of $E(\rho)$ using A14+UVII (WFF), and U-DDI (FP). The two sets of variational minima obtained at $\rho>0.16 \mathrm{fm}^{-3}$ are labeled LDP and HDP.

FIG. 3. Constrained energies: $E_{c}\left(\rho, d_{t} / r_{0}\right)=\frac{1}{2}\left(E_{P B}+E_{J F}\right)+\Lambda\left[\left(I_{c}-1\right)^{2}+\left(\frac{1}{3} I_{\tau}+1\right)^{2}\right]$, of SNM using A18+UIX. $\Lambda$ was set to $1000 \mathrm{MeV}$, in order to keep the integrals of the two-body densities $I_{c}$ and $I_{\tau}$ within $5 \%$ of their exact values of 1 and -3 during minimization.

FIG. 4. Constrained energies: $E_{c}\left(\rho, d_{t} / r_{0}\right)=\frac{1}{2}\left(E_{P B}+E_{J F}\right)+\Lambda\left(I_{c}-1\right)^{2}$ of PNM, using A18+UIX. $\Lambda$ was set to $1000 \mathrm{MeV}$.

FIG. 5. $\rho_{2}^{p=1-6}$ for SNM at $\rho=0.16 \mathrm{fm}^{-3}$

FIG. 6. $\rho_{T, S, M=0,1,0}^{(2)}(r ; \theta=0, \pi / 2)$ for SNM at $\rho=0.16 \mathrm{fm}^{-3}$, and for light nuclei scaled to match the maximum value of the SNM distribution.

FIG. 7. $\rho_{T, S, M=1,0,0}^{(2)}(r)$ for SNM at $\rho=0.16 \mathrm{fm}^{-3}$, and for light nuclei scaled to match the maximum value of the SNM distribution.

FIG. 8. Two-body densities $\rho_{T, S, M}^{(2)}$ for SNM at $\rho=0.36 \mathrm{fm}^{-3}$. The full and dashed lines represent results for the LDP and HDP, respectively

FIG. 9. Two-body densities $\rho_{S, M}^{(2)}$ for PNM at $\rho=0.2 \mathrm{fm}^{-3}$. The full and dashed lines represent results for the LDP and HDP, respectively

FIG. 10. $v^{\sigma \tau}(r)$ and $\tilde{v}^{\sigma}(r)$ in U14, A14 and A18 models of the nucleon-nucleon interaction. 
FIG. 11. $S_{L}(q)$ for SNM and PNM.

FIG. 12. $W_{L}(q) / S_{L}(q)$ for SNM and PNM. $q^{2} / 2 m$ (dash-dot curve) is plotted for comparison.

FIG. 13. $\xi(q)$ for SNM and PNM.

FIG. 14. Pion excess function, $\eta(q)$ for SNM and PNM.

FIG. 15. (a)3-body separable direct, (b)3-body separable, interacting exchange, (c)3-body separable, passive exchange, (d) direct central chain, (e) interacting exchange central chain (f) pair exchange central chain and (g) circular exchange central chain diagrams. 


\section{TABLES}

TABLE I. Contributions to the Energy of SNM in MeV

\begin{tabular}{|c|c|c|c|c|c|c|}
\hline & U14 & U14 & A18 & A18 & A18+IX & A18+IX \\
\hline$k_{f}\left(\mathrm{fm}^{-1}\right)$ & 1.33 & 1.60 & 1.33 & 1.60 & 1.33 & 1.60 \\
\hline$\rho \quad\left(\mathrm{fm}^{-3}\right)$ & .16 & .28 & .16 & .28 & .16 & .28 \\
\hline$d_{c}(\mathrm{fm})$ & 2.15 & 1.79 & 2.13 & 2.08 & 1.80 & 1.67 \\
\hline$d_{t}(\mathrm{fm})$ & 3.44 & 2.86 & 5.67 & 4.76 & 4.81 & 3.81 \\
\hline$\alpha$ & .80 & .80 & .65 & .61 & .80 & .90 \\
\hline$\langle T\rangle-1 \mathrm{~B}$ & 22.01 & 31.85 & 22.01 & 31.85 & 22.01 & 31.85 \\
\hline$\langle v\rangle-2 \mathrm{~B}-\mathrm{s}$ & -56.40 & -75.21 & -66.17 & -100.68 & -66.41 & -105.34 \\
\hline$\langle T\rangle-2 \mathrm{~B}-\mathrm{s}$ & 16.08 & 21.72 & 19.01 & 28.51 & 20.25 & 33.47 \\
\hline$\langle v\rangle-2 \mathrm{~B}-\mathrm{b}$ & -3.23 & -6.37 & -2.29 & -5.02 & -2.38 & -5.76 \\
\hline$\langle T\rangle-2 \mathrm{~B}-\mathrm{b}$ & .80 & 1.50 & .54 & 1.10 & .62 & 1.58 \\
\hline$\langle v\rangle-2 \mathrm{~B}-\mathrm{q}$ & 1.11 & 3.56 & 4.31 & 11.39 & 4.46 & 12.44 \\
\hline$\langle v+T\rangle-2 \mathrm{~B}$ & -41.64 & -54.80 & -44.61 & -64.71 & -43.46 & -63.61 \\
\hline$\langle v+T\rangle-\mathrm{MB}-\mathrm{s}$ & 4.76 & 2.91 & 6.47 & 9.31 & 5.50 & 8.47 \\
\hline$\langle v+T\rangle-\mathrm{MB}-\mathrm{b}$ & -.21 & -1.02 & -.28 & -1.01 & -.22 & -.72 \\
\hline$\langle v\rangle-\mathrm{MB}-\mathrm{q}$ & 1.61 & 3.91 & 3.00 & 8.54 & 3.38 & 10.62 \\
\hline$\left\langle V^{2 \pi}\right\rangle$ & .00 & .00 & .00 & .00 & -3.60 & -9.96 \\
\hline$\left\langle V^{R}\right\rangle$ & .00 & .00 & .00 & .00 & 6.33 & 22.09 \\
\hline$\delta E_{2 B}$ & -0.95 & -2.06 & -1.30 & -2.35 & -1.89 & -4.07 \\
\hline$\frac{1}{2}\left(E_{P B}+E_{J F}\right)+\delta E_{2 B}$ & -14.42 & -19.22 & -14.71 & -18.37 & -11.96 & -5.33 \\
\hline$\frac{1}{2}\left(E_{P B}-E_{J F}\right)$ & .30 & .27 & .55 & .82 & .60 & 1.10 \\
\hline
\end{tabular}


TABLE II. Contributions to $E\left(\rho, d_{t} / r_{0}\right)$ of SNM for A18+IX model in MeV

\begin{tabular}{|c|c|c|c|c|c|}
\hline$\rho\left(\mathrm{fm}^{-3}\right)$ & .16 & .28 & .28 & .36 & .36 \\
\hline$d_{t} / r_{0}$ & 4.20 & 4.00 & 6.00 & 4.00 & 6.00 \\
\hline$d_{c} / d_{t}$ & .38 & .41 & .25 & .44 & .28 \\
\hline$\alpha$ & .80 & .90 & .96 & .98 & .88 \\
\hline$\langle T\rangle-1 \mathrm{~B}$ & 22.11 & 32.10 & 32.10 & 37.96 & 37.96 \\
\hline$\langle v+T\rangle-2 \mathrm{~B}$ & -43.65 & -62.71 & -67.24 & -74.37 & -79.54 \\
\hline$\langle v+T\rangle-\mathrm{MB}$ & 8.71 & 18.11 & 35.29 & 28.52 & 43.70 \\
\hline$\langle T\rangle$ & 42.26 & 67.41 & 72.28 & 85.16 & 85.97 \\
\hline$\langle v\rangle$ & -55.09 & -79.91 & -72.13 & -93.06 & -83.85 \\
\hline$\left\langle v^{t \tau}\right\rangle$ & -29.14 & -45.82 & -49.65 & -56.94 & -59.23 \\
\hline$\left\langle v^{\sigma \tau}\right\rangle$ & -10.30 & -12.85 & -6.20 & -13.81 & -6.97 \\
\hline$\left\langle v^{c}\right\rangle$ & -26.04 & -47.27 & -47.10 & -61.65 & -61.25 \\
\hline$\left\langle V^{2 \pi}\right\rangle$ & -3.64 & -10.72 & -22.88 & -18.35 & -32.26 \\
\hline$\left\langle V^{R}\right\rangle$ & 6.42 & 22.65 & 23.76 & 40.69 & 41.71 \\
\hline$\delta E_{2 B}$ & -1.91 & -4.65 & -2.26 & -6.78 & -6.20 \\
\hline$\frac{1}{2}\left(E_{P B}+E_{J F}\right)+\delta E_{2 B}$ & -11.96 & -5.22 & -1.22 & 7.67 & 5.37 \\
\hline$\frac{1}{2}\left(E_{P B}-E_{J F}\right)$ & .60 & 1.05 & 2.30 & 1.45 & 2.82 \\
\hline
\end{tabular}


TABLE III. Expectation values of the Pion Exchange Interaction and Pion Excess Operators

\begin{tabular}{|c|c|c|c|}
\hline & $\left\langle v^{\pi}\right\rangle / A, \mathrm{MeV}$ & $\left\langle V^{2 \pi}\right\rangle / A, \mathrm{MeV}$ & $\left\langle\delta n_{\pi}^{(1)}\right\rangle / A$ \\
\hline $\mathrm{SNM}, \rho=0.16 \mathrm{fm}^{-3}$ & -31.53 & -3.64 & 0.05 \\
\hline $\mathrm{SNM}, \rho=0.36 \mathrm{fm}^{-3}, \mathrm{LDP}$ & -60.62 & -18.35 & 0.09 \\
\hline $\mathrm{SNM}, \rho=0.36 \mathrm{fm}^{-3}, \mathrm{HDP}$ & -66.86 & -32.26 & 0.15 \\
\hline $\mathrm{PNM}, \rho=0.16 \mathrm{fm}^{-3}$ & -0.67 & 1.23 & -0.01 \\
\hline PNM, $\rho=0.20 \mathrm{fm}^{-3}, \mathrm{LDP}$ & 0.14 & 1.90 & -0.01 \\
\hline PNM, $\rho=0.20 \mathrm{fm}^{-3}, \mathrm{HDP}$ & -18.20 & -8.67 & 0.05 \\
\hline
\end{tabular}

TABLE IV. MB-q Contributions in MeV to SNM at $\rho=0.28 \mathrm{fm}^{-3}$ using U14 and A18 NN-interactions

\begin{tabular}{lcr}
\hline \hline diagram & $\mathrm{U} 14$ & $\mathrm{~A} 18$ \\
\hline 2B-dir & 4.18 & 9.12 \\
2B-ex & -0.62 & 2.27 \\
$(\mathrm{a})$ & 3.47 & 8.57 \\
$(\mathrm{~b})$ & 0.22 & -1.54 \\
$(\mathrm{c})$ & 0.21 & 0.46 \\
$(\mathrm{~d}+\mathrm{f})$ & 0.20 & 0.49 \\
$(\mathrm{e}+\mathrm{g})$ & -0.19 & 0.56 \\
\hline \hline
\end{tabular}


TABLE V. Contributions to $\delta E_{2}$ from $l, S, J$ channels for A18+IX model in $\mathrm{MeV}$

\begin{tabular}{lccccccccc}
\hline \hline$\rho\left(\mathrm{fm}^{-3}\right)$ & ${ }^{1} S_{0}$ & ${ }^{1} P_{1}$ & ${ }^{1} D_{2}$ & ${ }^{1} F_{3}$ & ${ }^{3} P_{0}$ & ${ }^{3} P_{1}$ & ${ }^{3} D_{2}$ & ${ }^{3} F_{3}$ & ${ }^{3} P_{2}-{ }^{3} F_{2}$ \\
\hline 0.16 (SNM LDP) & 0.00 & 0.00 & -0.01 & 0.00 & -0.20 & -0.80 & -0.15 & 0.00 & -0.94 \\
0.28 (SNM LDP) & -0.03 & 0.00 & -0.03 & 0.00 & 0.00 & -1.74 & -0.24 & 0.00 & -2.16 \\
0.36 (SNM HDP) & -0.05 & 0.00 & -0.05 & 0.00 & -0.14 & -2.97 & -0.35 & 0.00 & -2.80 \\
0.16 (PNM LDP) & 0.00 & & -0.01 & & -0.15 & -0.62 & & 0.00 & -0.70 \\
0.20 (PNM LDP) & 0.00 & & -0.01 & & -0.16 & -0.83 & & -0.01 & -0.15 \\
0.24 (PNM HDP) & -0.22 & & -0.09 & -0.41 & -1.10 & & 0.00 & -0.20 \\
\hline \hline
\end{tabular}




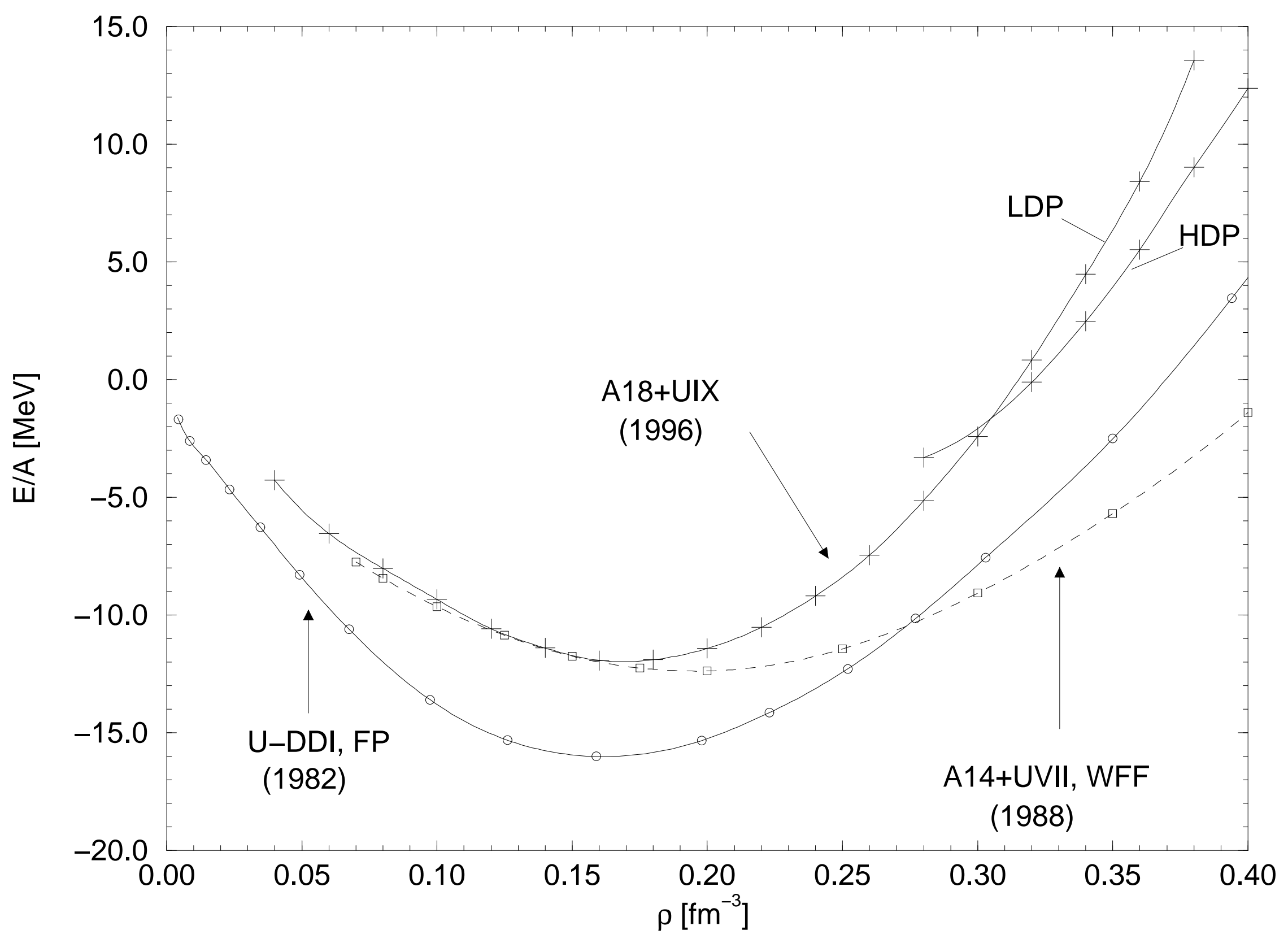




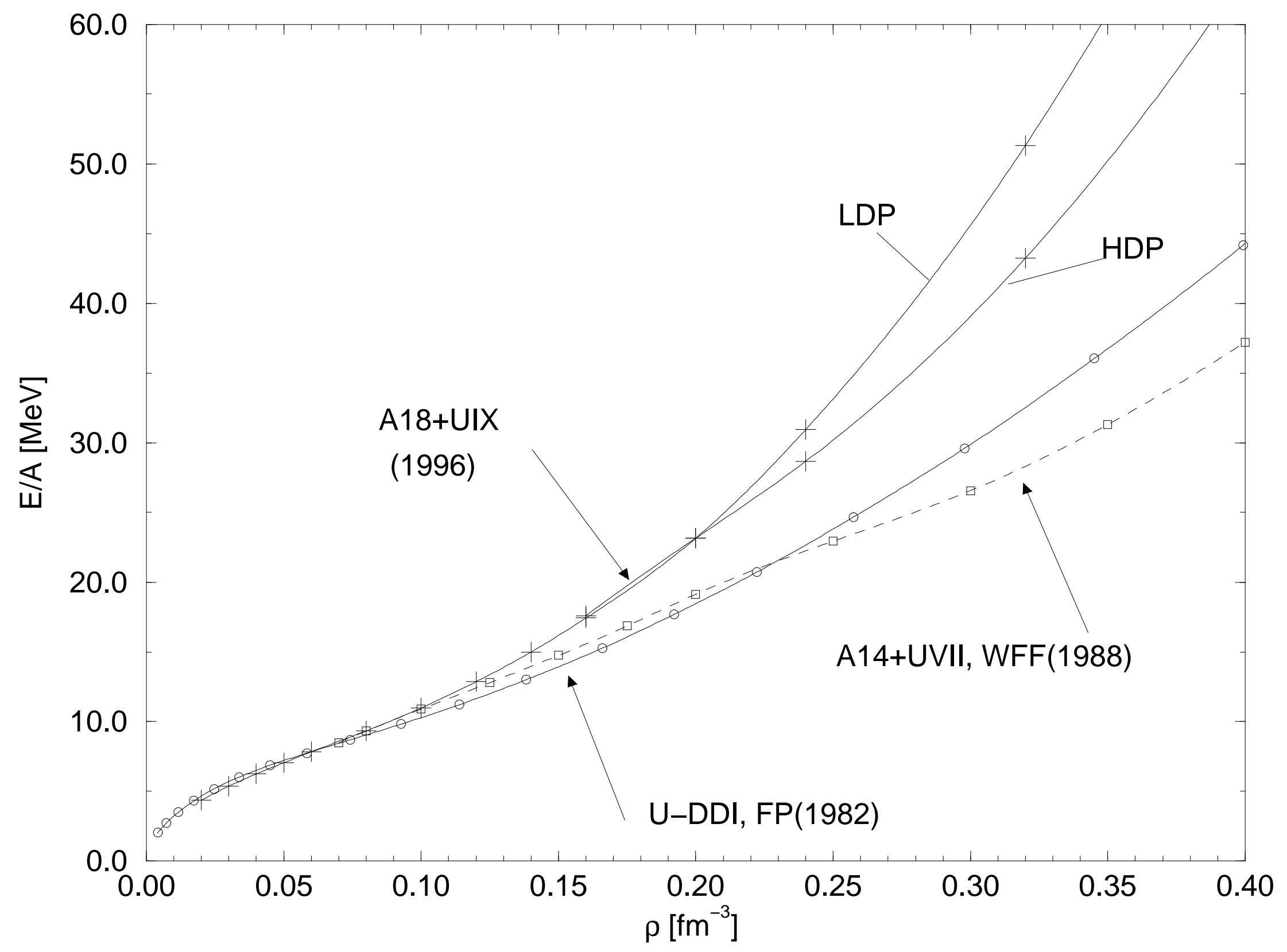




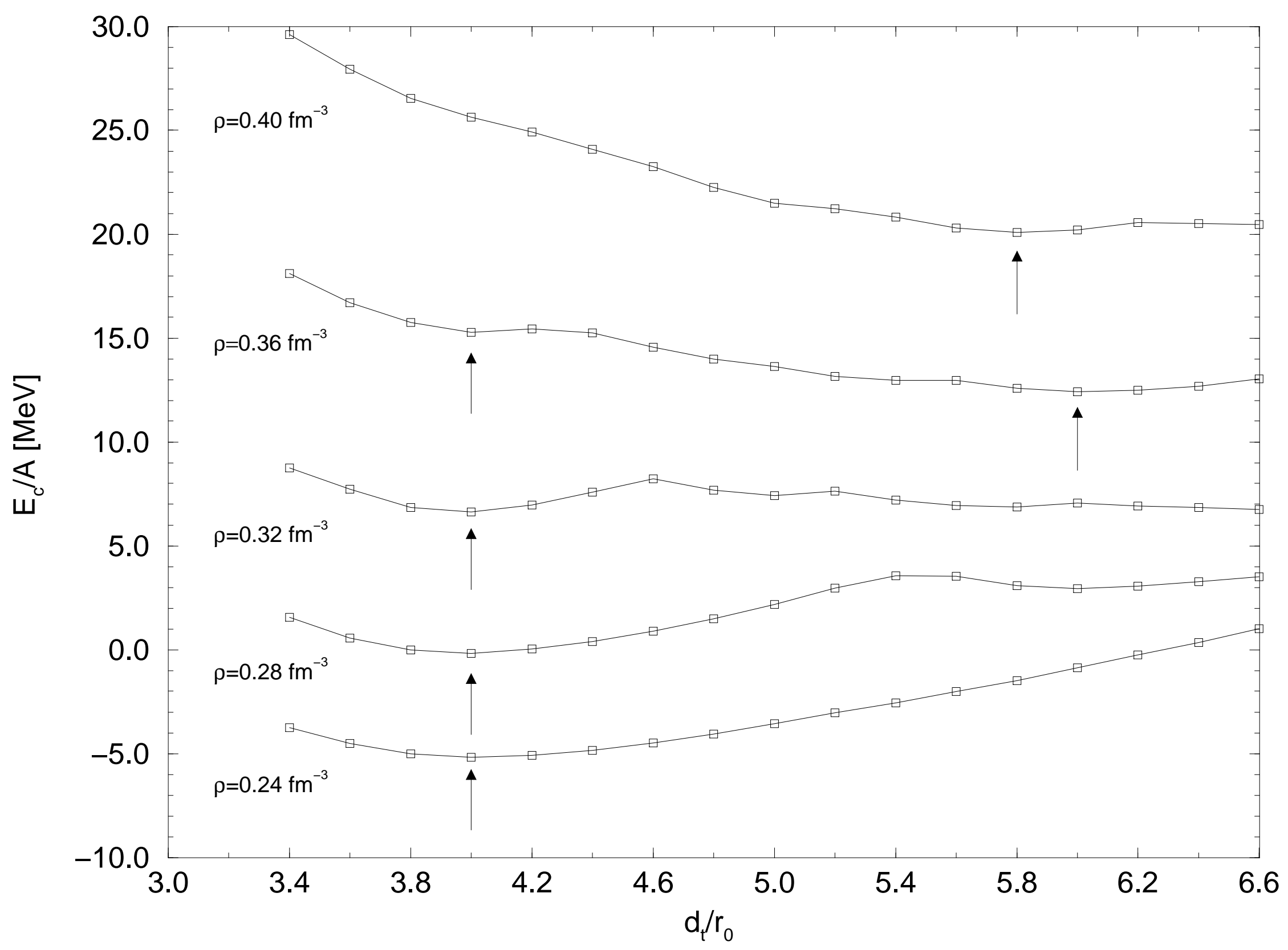




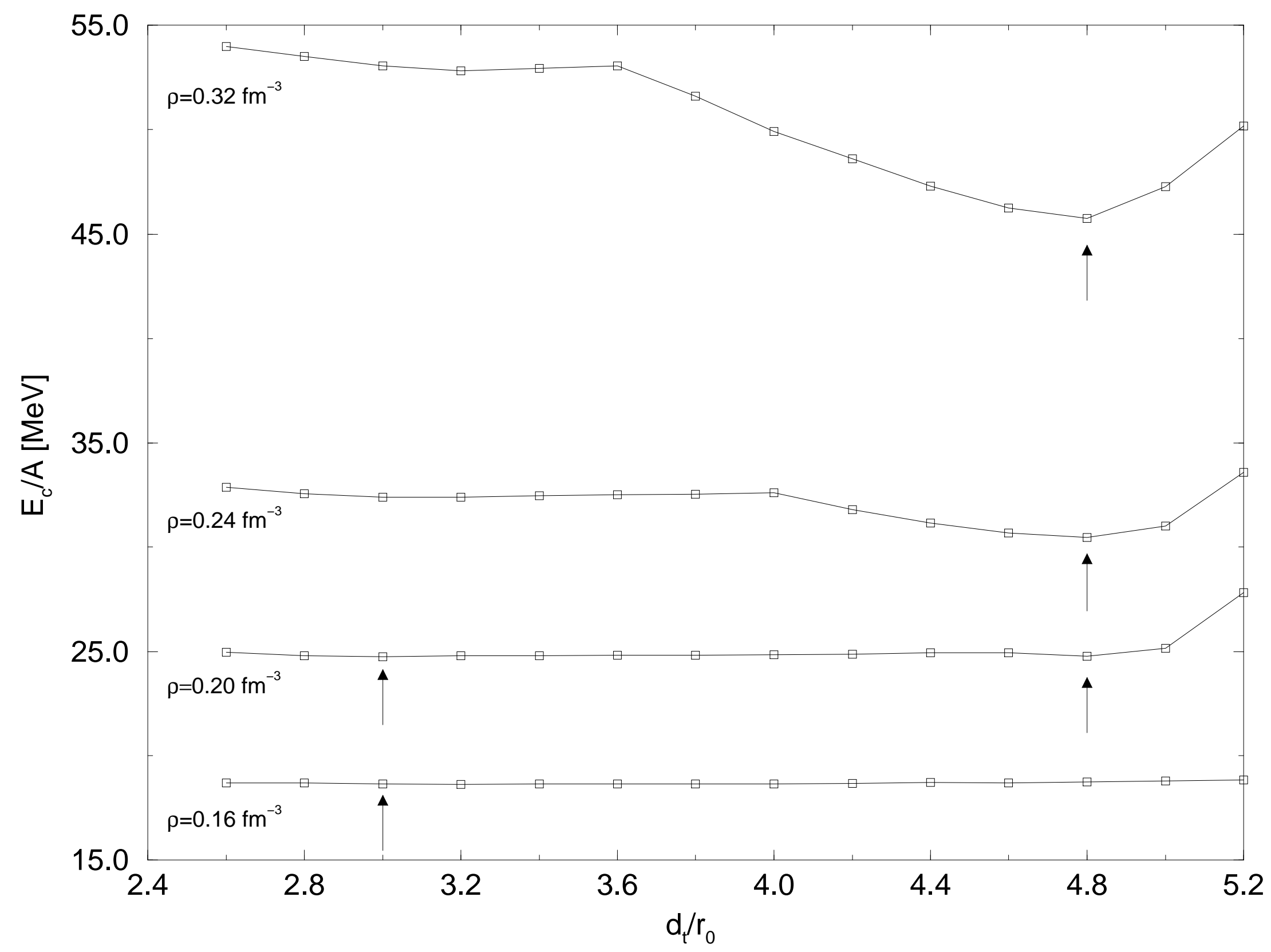




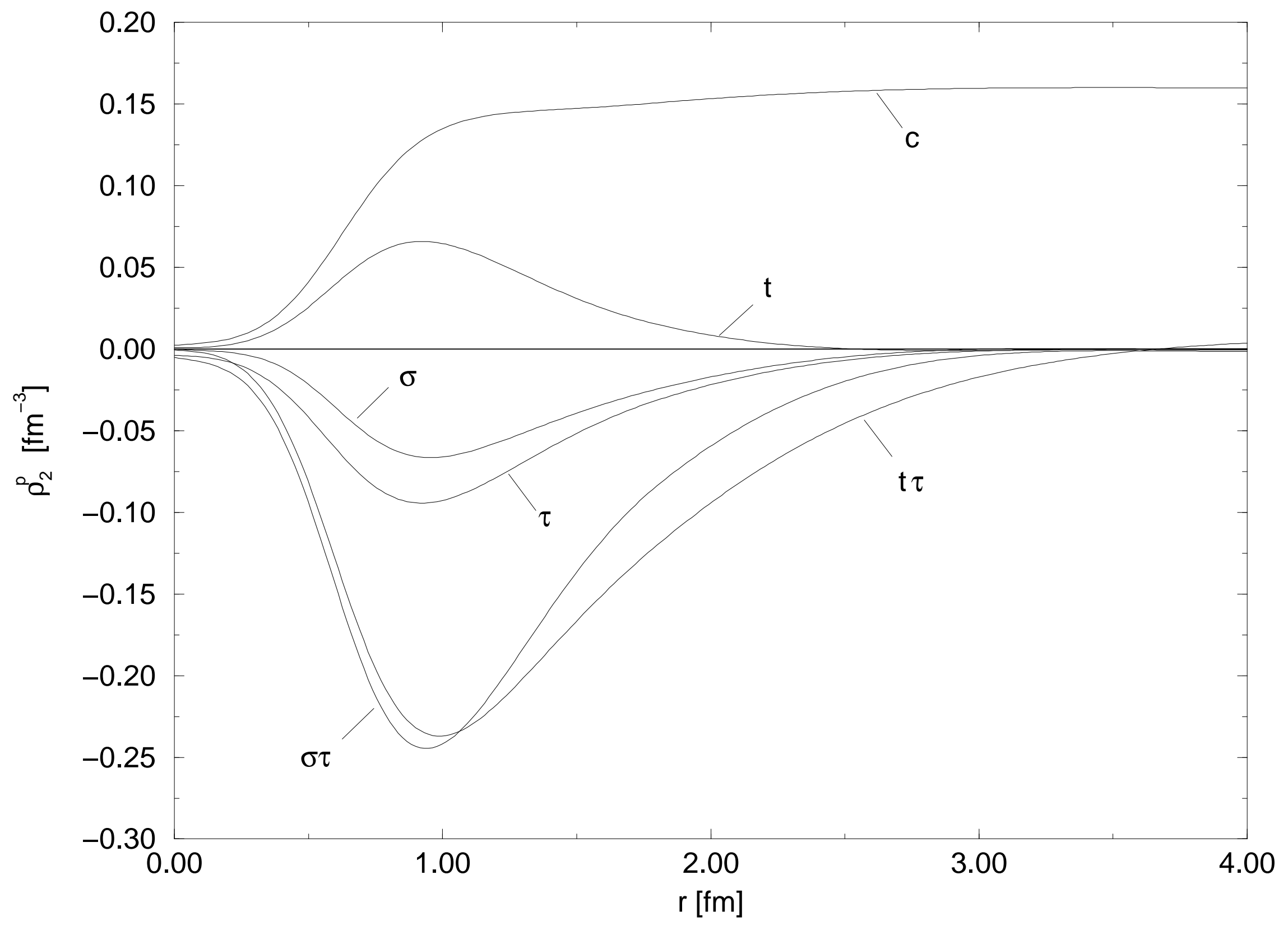




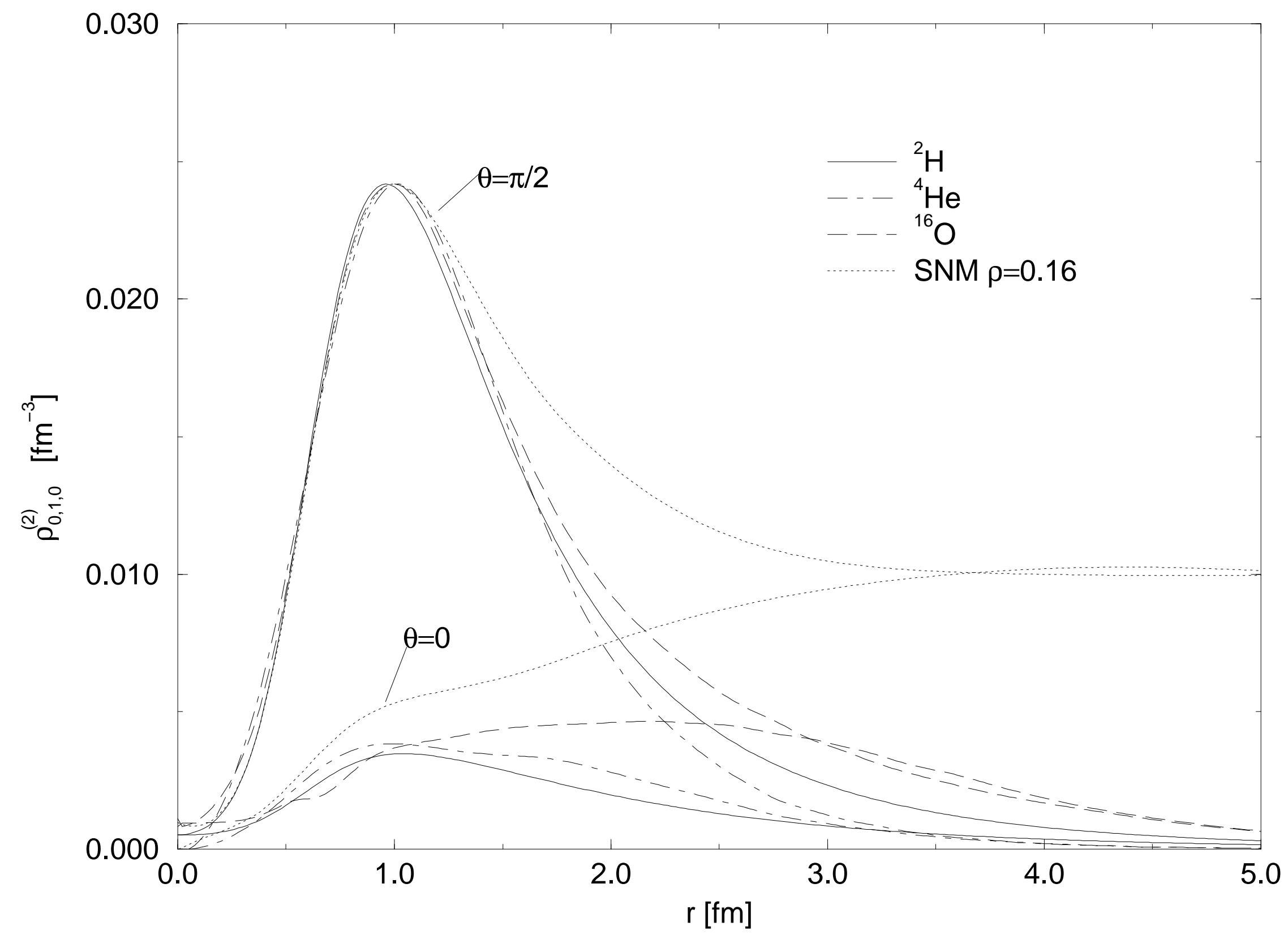




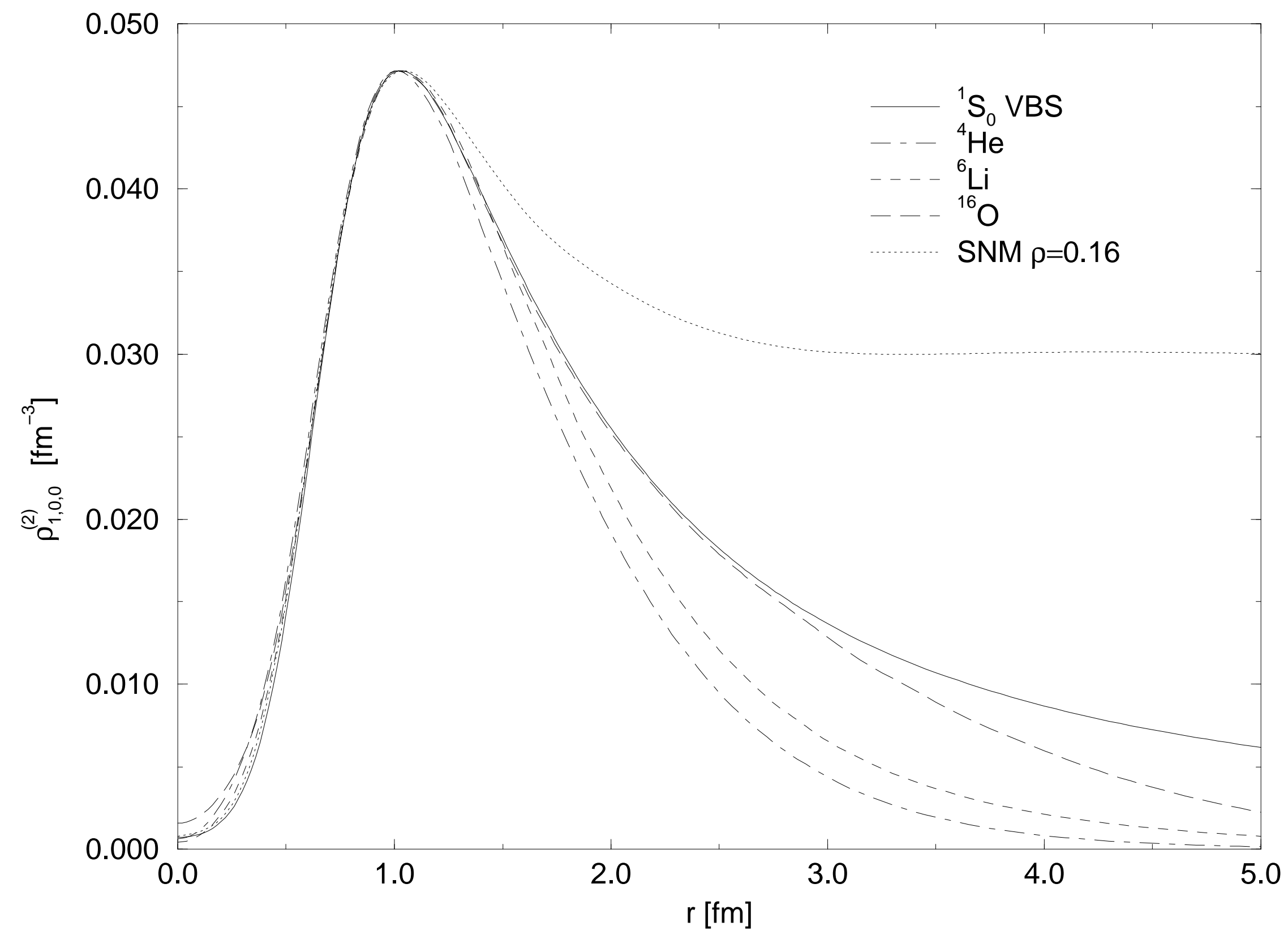




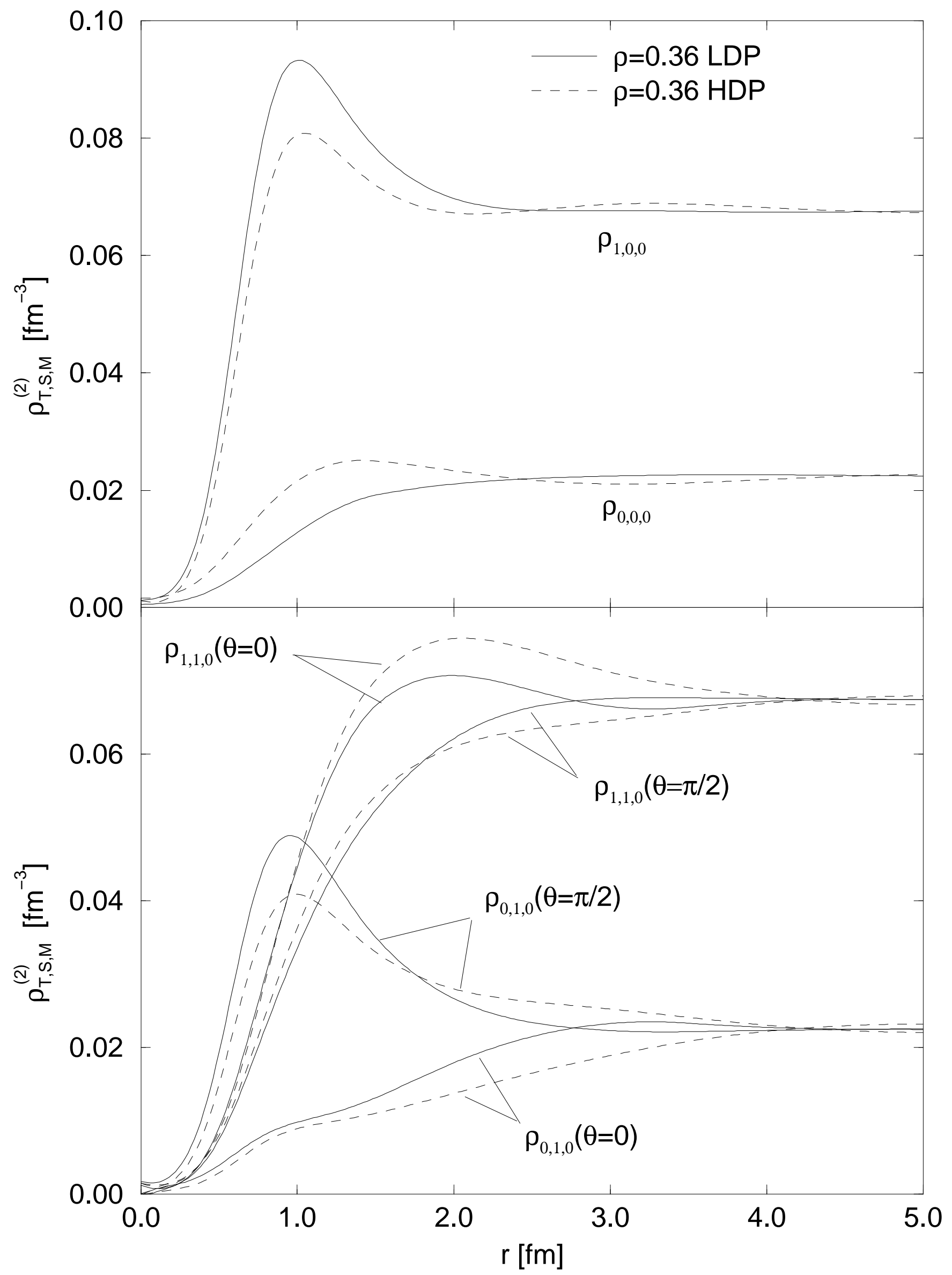




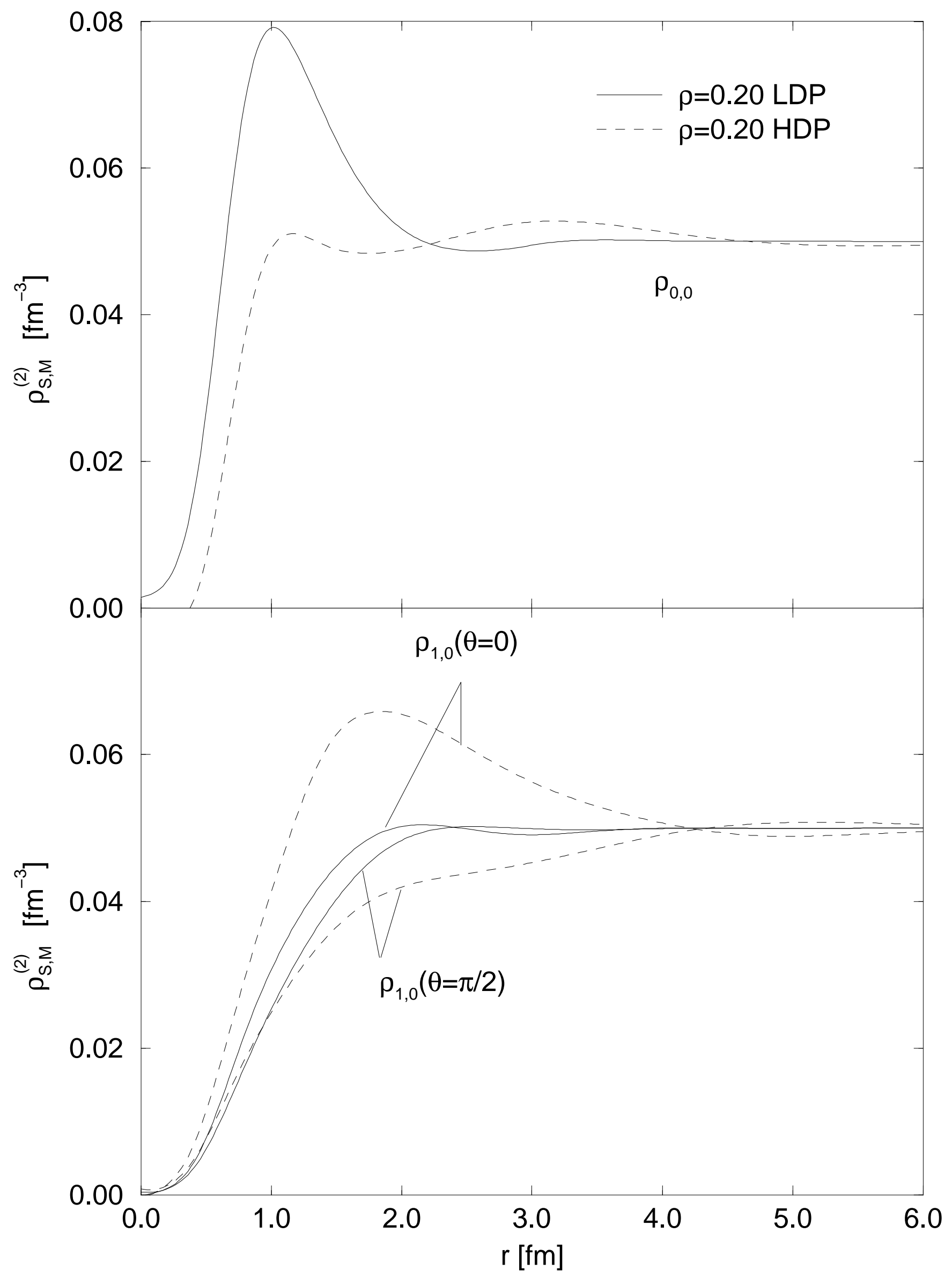




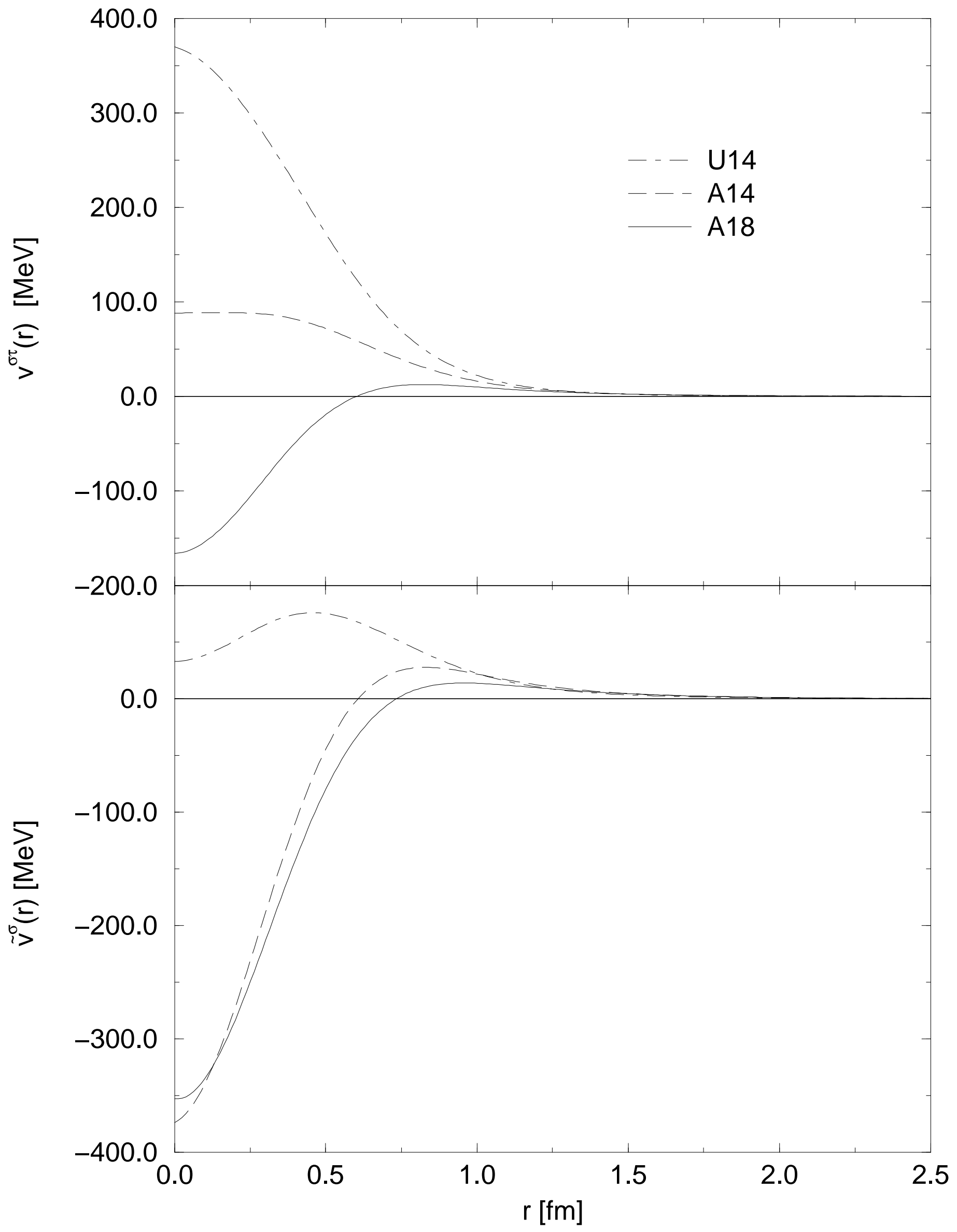




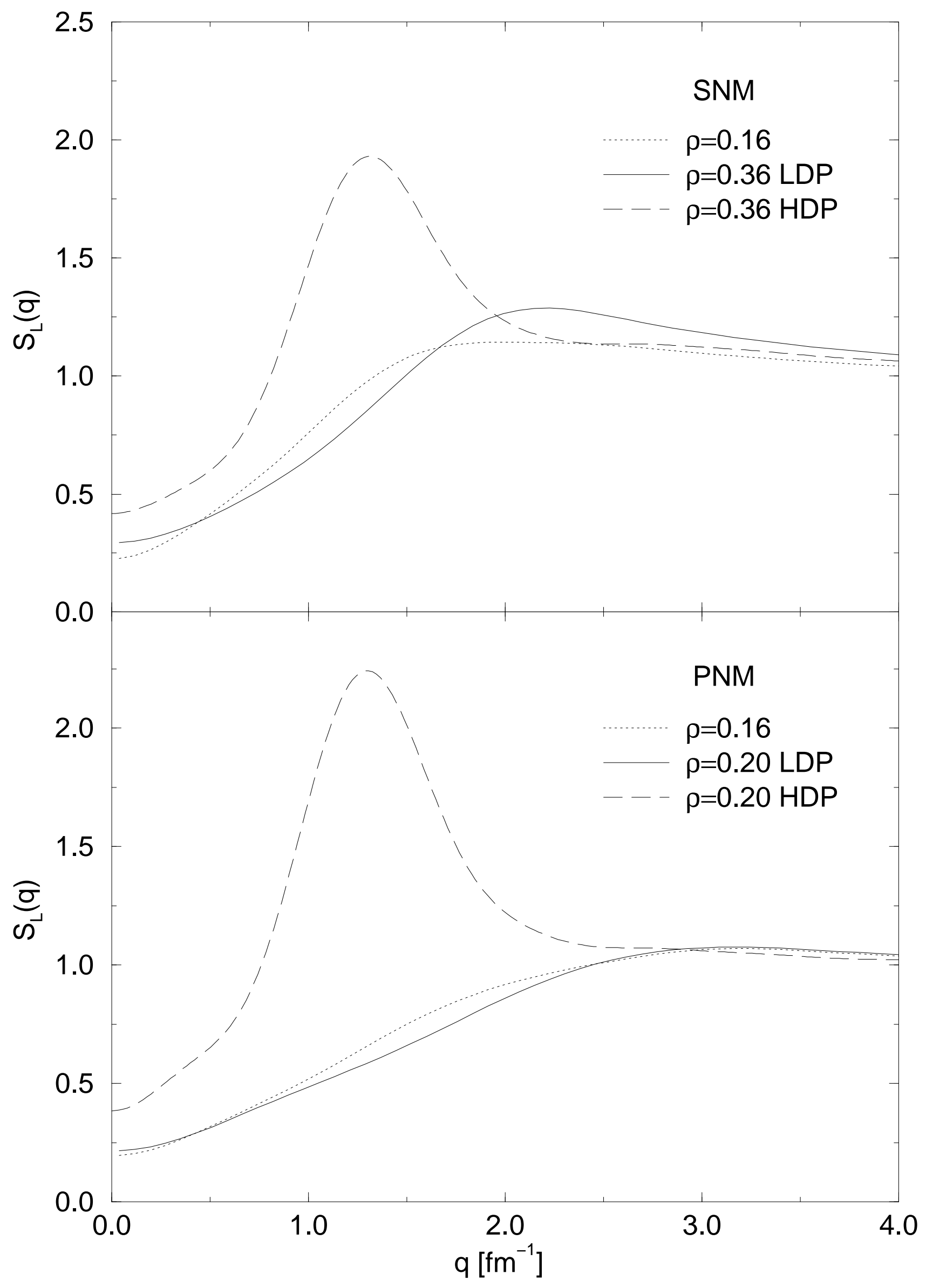




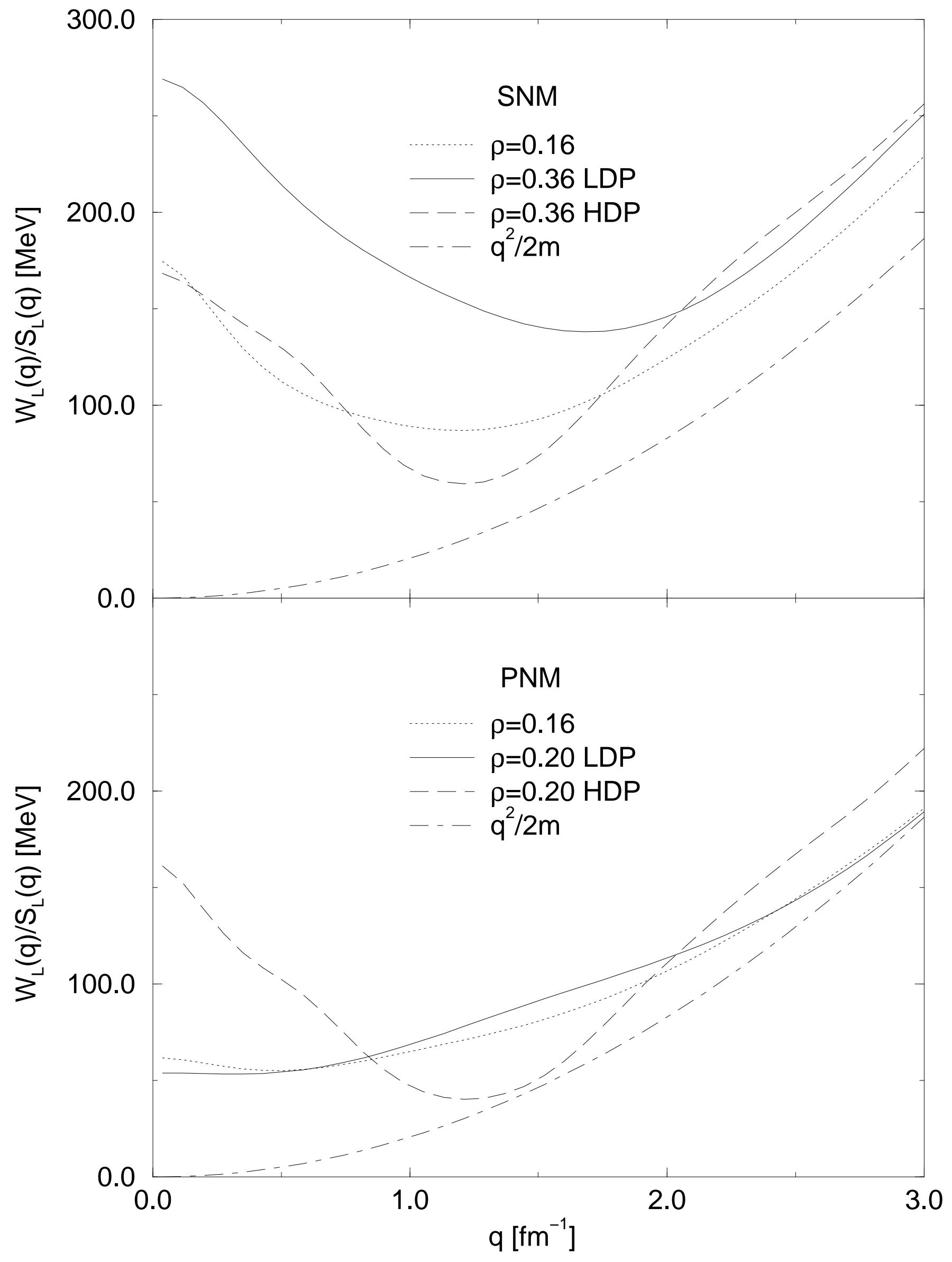




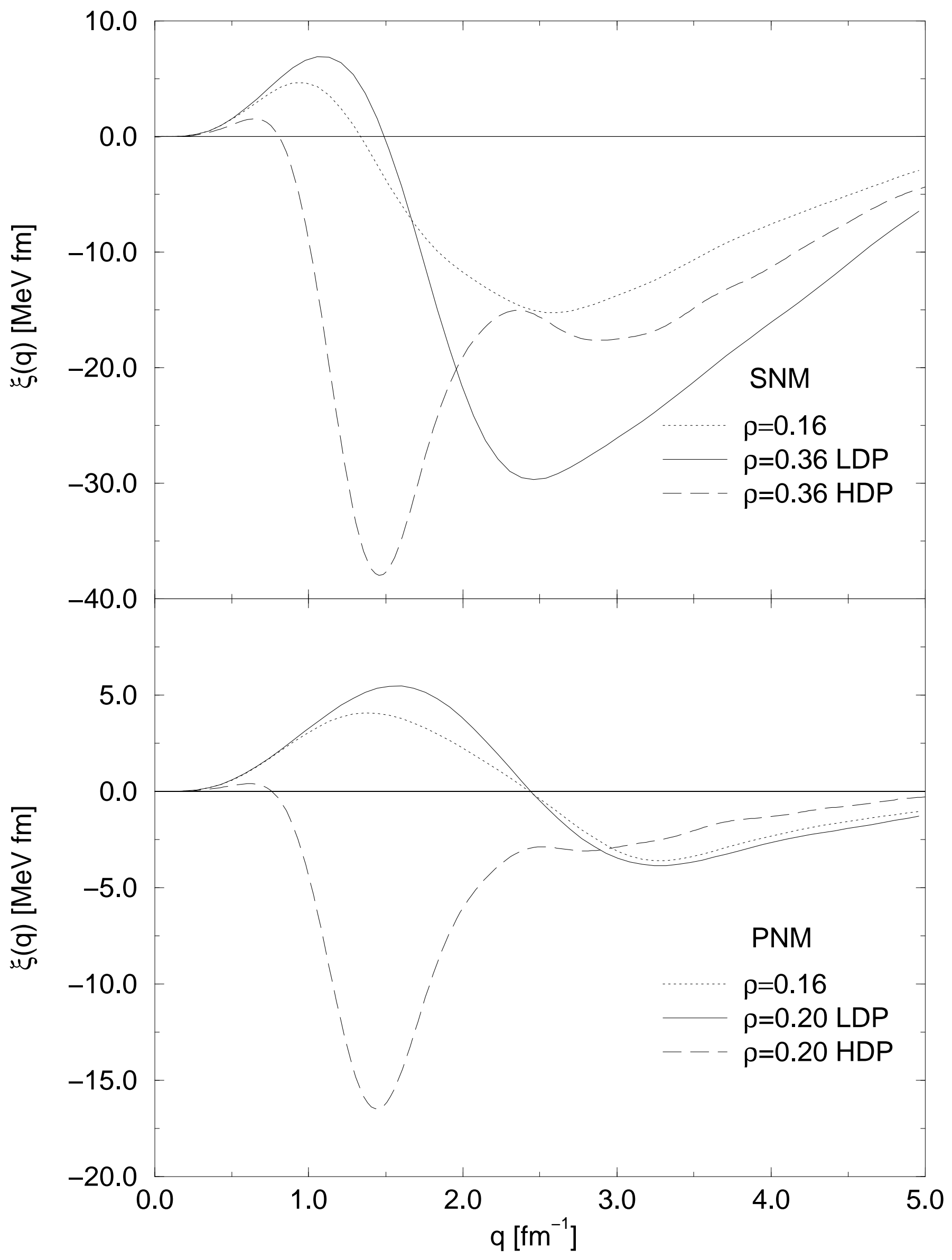




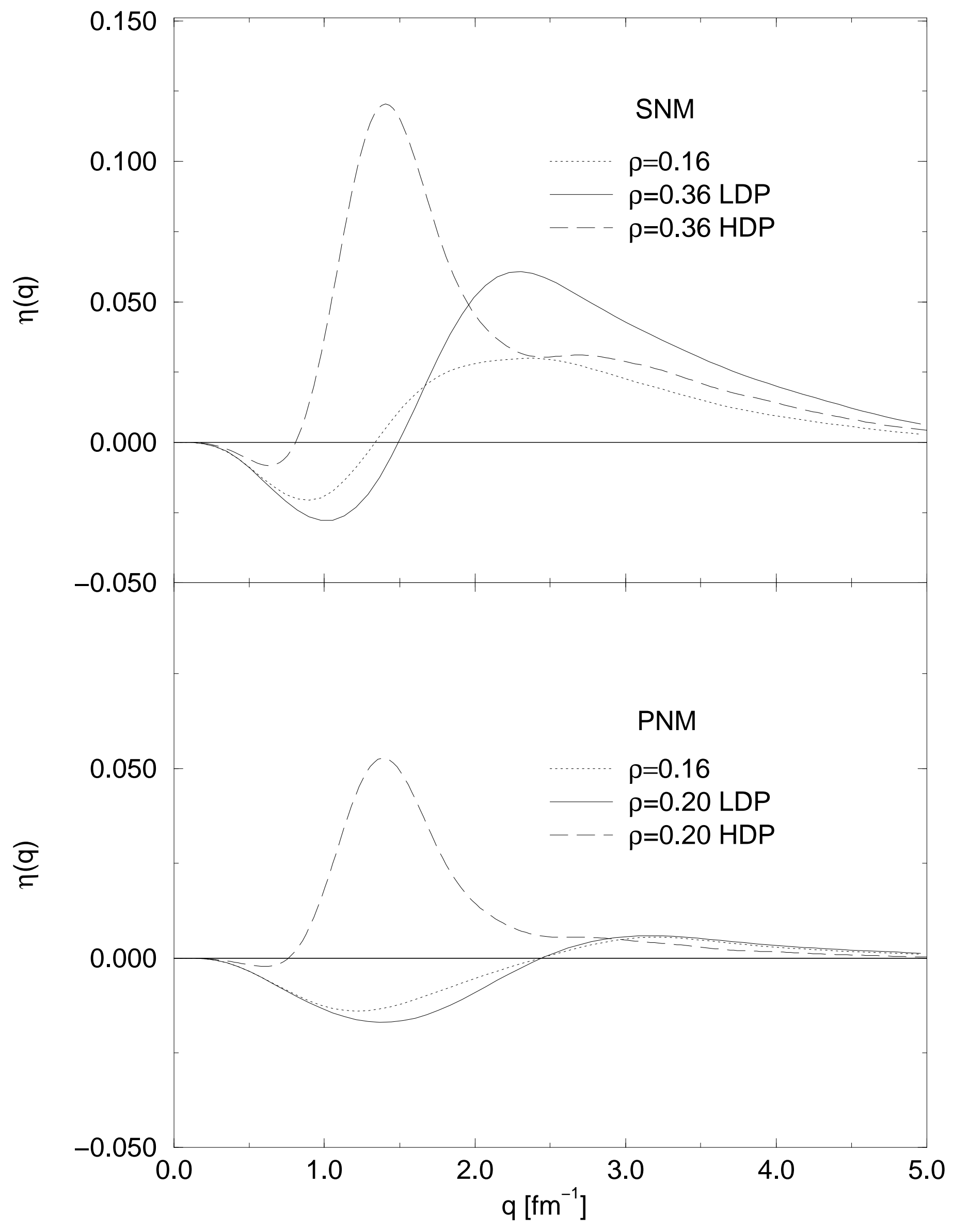




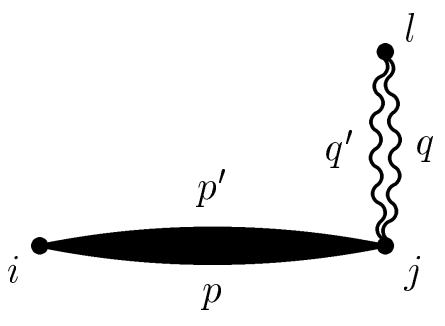

(a)

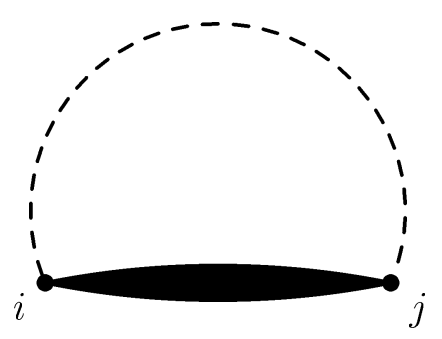

(d)

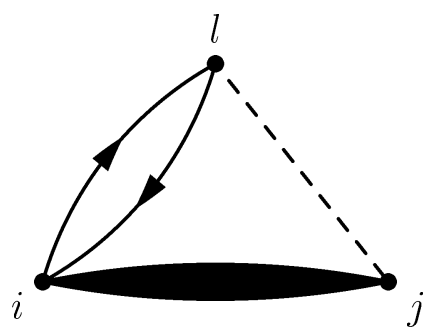

(f)

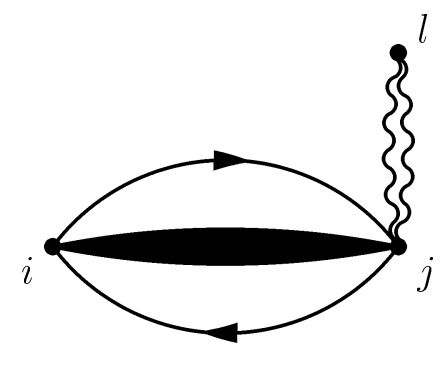

(b)

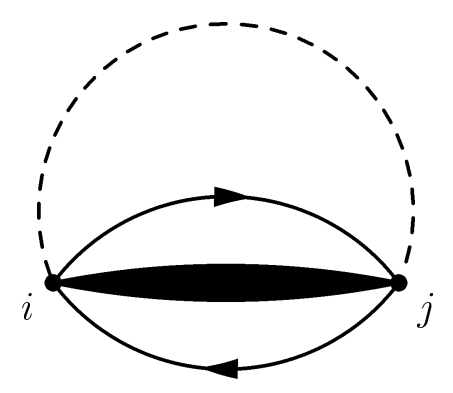

(e)

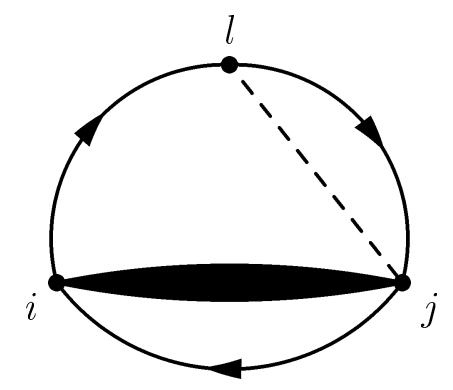

(g)

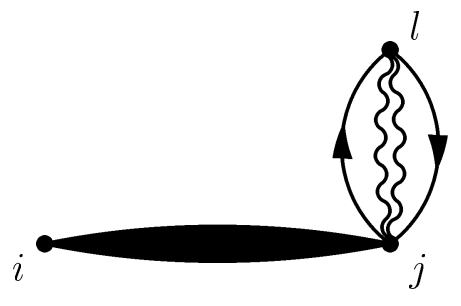

(c) 